\title{
SECOND-ORDER INVARIANT DOMAIN PRESERVING APPROXIMATION OF THE EULER EQUATIONS USING CONVEX LIMITING*
}

\author{
JEAN-LUC GUERMOND ${ }^{\dagger}$, MURTAZO NAZAROV $\ddagger$, BOJAN POPOV ${ }^{\dagger}$, AND IGNACIO \\ TOMAS $^{\dagger}$
}

\begin{abstract}
A new second-order method for approximating the compressible Euler equations is introduced. The method preserves all the known invariant domains of the Euler system: positivity of the density, positivity of the internal energy and the local minimum principle on the specific entropy. The technique combines a first-order, invariant domain preserving, Guaranteed Maximum Speed method using a Graph Viscosity (GMS-GV1) with an invariant domain violating, but entropy consistent, high-order method. Invariant domain preserving auxiliary states, naturally produced by the GMS-GV1 method, are used to define local bounds for the high-order method which is then made invariant domain preserving via a convex limiting process. Numerical tests confirm the second-order accuracy of the new GMS-GV2 method in the maximum norm, where 2 stands for second-order. The proposed convex limiting is generic and can be applied to other approximation techniques and other hyperbolic systems.
\end{abstract}

Key words. hyperbolic systems, Riemann problem, invariant domain, entropy inequality, highorder method, exact rarefaction, quasiconvexity, limiting, finite element method.

AMS subject classifications. 65M60, 65M12, 35L65, 35L45

1. Introduction. The objective of the present work is to present an approximation technique for the compressible Euler equations that is explicit in time, secondorder accurate in space and time, and invariant domain preserving. The method is presented in the context of continuous finite elements, but it is quite general and can be applied to other discretization settings like discontinuous Galerkin and finite volume techniques. Like in many other high-order approximation methods, the proposed technique consists of combining a first-order, invariant domain preserving, and entropy satisfying approximation with a high-order entropy consistent approximation. The high-order method is made invariant domain preserving and formally entropy compliant by adapting the artificial viscosity through a limitation process. One key novelty is that the density, the internal energy and the specific entropy are limited by using bounds that are in the domain of dependence of the local data. Another novelty is that the limiting bounds are local for all the quantities, and these bounds are naturally satisfied by the low-order solution.

The so-called Flux Transport Corrected method (FCT) introduced in Boris and Book [4] for approximating the one-dimensional mass conservation equation and later generalized to multi dimensions in Zalesak [45] are among the first successful techniques to produce second-accuracy while imposing pointwise bounds such as positivity of the density. This methodology can be used to preserve the maximum principle for any scalar conservation equation. We refer the reader to Kuzmin and Turek

*This material is based upon work supported in part by the National Science Foundation grants DMS-1619892 and DMS-1620058, by the Air Force Office of Scientific Research, USAF, under grant/contract number FA9550-15-1-0257, and by the Army Research Office under grant/contract number W911NF-15-1-0517.

Sept. 30th 2017. Submitted for publication to SIAM SISC.

${ }^{\dagger}$ Department of Mathematics, Texas A\&M University 3368 TAMU, College Station, TX 77843, USA.

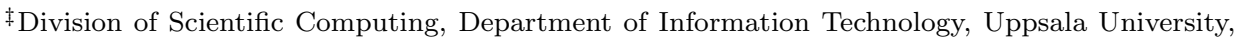
Uppsala, Sweden 
[28], Kuzmin et al. [29] for reviews on FCT. Unfortunately, the FCT algorithm, as proposed in the above references, is not well suited to enforce constraints that are not affine. For instance it cannot be (easily) applied to guarantee the positivity of the specific internal energy and the minimum principle on the specific entropy since these are quasiconcave constraints. (The said constraints can be made concave by multiplication by the density as it is shown in $\S 4$.) In the context of finite volumes, efficient second-order limitation techniques for the specific internal energy and the specific entropy have been first proposed in Khobalatte and Perthame [25], Perthame and Qiu [35], and Perthame and Shu [36]. These ideas have been extended to Discontinuous Galerkin framework in a series of papers by Zhang and Shu [47, 48], Jiang and Liu [24]. The key argument that is common to [25, 36, 35] is to rely on convex combinations and concavity. In the present paper we are going to build on these ideas and propose a general FCT-like post-processing methodology to enforce general concave constraints for the Euler equations. Instead of limiting slopes or reconstructed approximations, we adopt an algebraic point of view similar to FCT. The method is presented in the context of continuous finite elements, but since it is algebraic, it can be applied to finite volumes and discontinuous Galerkin approximation techniques as well.

The paper is organized as follows. The problem is formulated in $\S 2$. The finite element setting and the notation are also introduced in this section. The time and space approximation using continuous finite elements is described in $\S 3$. Both the first-order, invariant domain preserving, entropy satisfying method and the high-order invariant domain violating method are detailed in this section. The bulk of the novel material is reported in $\S 4$. The main results of this section are the local bounds given in (4.1)-(4.4) together with Lemma 4.3 and Lemma 4.4. The performance of the proposed method is illustrated in $\S 5$.

2. Preliminaries. We introduce in this section the Euler equations and the finite element setting. Some important properties of the Euler equations that are used later in the paper are also recalled. The reader who is familiar with the Euler equations, invariant domains, and the finite element theory is invited to jump to $\S 3$.

2.1. The Euler equations. Let $d$ be the space dimension and $D$ be an open polyhedral domain in $\mathbb{R}^{d}$. We consider the compressible Euler equations in conservative form in $\mathbb{R}^{d}$ :

$$
\begin{aligned}
& \partial_{t} \rho+\nabla \cdot \boldsymbol{m}=0, \\
& \partial_{t} \boldsymbol{m}+\nabla \cdot(\boldsymbol{v} \otimes \boldsymbol{m})+\nabla p=0, \\
& \partial_{t} E+\nabla \cdot(\boldsymbol{v}(E+p))=0, \\
& \rho(\boldsymbol{x}, 0)=\rho_{0}, \quad \boldsymbol{m}(\boldsymbol{x}, 0)=\boldsymbol{m}_{0}, \quad E(\boldsymbol{x}, 0)=E_{0} .
\end{aligned}
$$

The independent variables are $(\boldsymbol{x}, t) \in D \times \mathbb{R}_{+}$. The dependent variables, henceforth called conservative variables, are the density, $\rho$, the momentum, $\boldsymbol{m}$ and the total energy, $E$. The quantity $\boldsymbol{v}:=\rho^{-1} \boldsymbol{m}$ is the velocity of the fluid particles. The pressure, $p$, is given by the equation of state which we assume to be derived from a specific entropy, $s: \mathbb{R}_{+} \times \mathbb{R}_{+} \rightarrow \mathbb{R}$, defined through the thermodynamics identity: $T \mathrm{~d} s:=\mathrm{d} e-p \rho^{-2} \mathrm{~d} \rho$, where $e:=\rho^{-1} E-\frac{1}{2} \boldsymbol{v}^{2}$ is the specific internal energy. For instance it is common to take $s(\rho, e)-s_{0}=\log \left(e^{\frac{1}{\gamma-1}} \rho^{-1}\right)$ for a polytropic ideal gas. Using the notation $s_{e}(\rho, e):=\frac{\partial s}{\partial e}(\rho, e)$ and $s_{\rho}(\rho, e):=\frac{\partial s}{\partial \rho}(\rho, e)$, the equation of state then takes the form $p:=-\rho^{2} s_{\rho} s_{e}^{-1}$. To simplify the notation we introduce the 
conservative variable $\boldsymbol{u}:=(\rho, \boldsymbol{m}, E)^{\top} \in \mathbb{R}^{d+2}$ and the flux $\boldsymbol{f}(\boldsymbol{u}):=(\boldsymbol{m}, \boldsymbol{v} \otimes \boldsymbol{m}+$ $p \mathbb{I}, \boldsymbol{v}(E+p))^{\top} \in \mathbb{R}^{m \times(d+2)}$, where $\mathbb{I}$ is the $d \times d$ identity matrix. To avoid an abuse of notation and ambiguities, we introduce

$$
\Phi(\boldsymbol{u}):=s(\rho, e(\boldsymbol{u}))
$$

where $e(\boldsymbol{u}):=\rho^{-1} E-\frac{\boldsymbol{m}^{2}}{2 \rho}, \boldsymbol{m}^{2}:=\|\boldsymbol{m}\|_{\ell^{2}}^{2}$ and $\|\cdot\|_{\ell^{2}}$ is the Euclidean norm, i.e., $\Phi$ is the specific entropy expressed as a function of the conservative variables. Finally, we call internal energy the quantity $\varepsilon:=\rho e=E-\frac{1}{2} \rho \boldsymbol{v}^{2}$.

The convention adopted in this paper is that for any vectors $\boldsymbol{a}, \boldsymbol{b}$, with entries $\left\{a_{k}\right\}_{k=1, \ldots, d},\left\{b_{k}\right\}_{k=1, \ldots, d}$, the following holds: $(\boldsymbol{a} \otimes \boldsymbol{b})_{k l}=a_{k} b_{l}$ and $\nabla \cdot \boldsymbol{a}=$ $\sum_{k=1, \ldots, d} \partial_{x_{k}} a_{k},(\nabla \boldsymbol{a})_{k l}=\partial_{x_{l}} a_{k} \cdot \boldsymbol{a} \cdot \nabla=\sum_{k=1, \ldots, d} a_{k} \partial_{x_{k}}$. Moreover, for any secondorder tensor $\mathrm{g}$, with entries $\left\{\mathrm{g}_{k l}\right\}_{k, l=1, \ldots, d}$, we define $(\nabla \cdot \mathrm{g})_{k}=\sum_{l=1, \ldots, d} \partial_{x_{l}} \mathrm{~g}_{k l}$, $(\mathrm{g} \boldsymbol{a})_{k}=\sum_{l=1, \ldots, d} \mathrm{~g}_{k l} a_{l},\left(\boldsymbol{a}^{\top} \mathrm{g}\right)_{l}=\sum_{l=1, \ldots, d} a_{k} \mathrm{~g}_{k l}$.

To avoid technicalities regarding boundary conditions, we assume that either periodic boundary conditions are enforced, or the initial data is compactly supported and in that case we are interested in the solution before the domain of influence of $\left(\rho_{0}, \boldsymbol{m}_{0}, E_{0}\right)$ reaches the boundary of $D$, i.e., homogeneous Dirichlet boundary conditions are enforced. (Some details are given in $\S 5.7$ on how to deal with realistic boundary conditions.)

2.2. Intrinsic properties. The well-posedness of (2.1) is an extremely difficult question that is far beyond the scope of the present paper. But to make sense of the approximation techniques to be presented in the rest of the paper we are going to rely on the notion of solution of one-dimensional Riemann problems which is more tractable. For any unit vector $\boldsymbol{n} \in \mathbb{R}^{d}$, we consider the following Riemann problem:

$$
\partial_{t} \boldsymbol{u}+\partial_{x}(\boldsymbol{f}(\boldsymbol{u}) \cdot \boldsymbol{n})=0, \quad(x, t) \in \mathbb{R} \times \mathbb{R}_{+}, \quad \boldsymbol{u}(x, 0)= \begin{cases}\boldsymbol{u}_{L}, & \text { if } x<0 \\ \boldsymbol{u}_{R}, & \text { if } x>0\end{cases}
$$

and assume that there exists a so-called admissible set $\mathcal{A}$ such that, for any pair of states $\left(\boldsymbol{u}_{L}, \boldsymbol{u}_{R}\right) \in \mathcal{A} \times \mathcal{A}$, this problem has a unique physical (entropy) solution henceforth denoted by $\boldsymbol{u}\left(\boldsymbol{n}, \boldsymbol{u}_{L}, \boldsymbol{u}_{R}\right)(x, t)$. This assumption holds true, at least for the covolume equation of state, with $\mathcal{A}:=\{\boldsymbol{u} \mid \rho>0, e>0\}$, and we refer the reader to Godlewski and Raviart [8, Thm. II.3.1] for a similar statement with more general equations of states. We now introduce notions of invariant sets and invariant domains. (Our definition is slightly different from those in Chueh et al. [6], Hoff [21], Frid [7].)

Definition 2.1 (Invariant set). We say that a set $B \subset \mathcal{A} \subset \mathbb{R}^{m}$ is invariant for (2.1) if for any pair $\left(\boldsymbol{u}_{L}, \boldsymbol{u}_{R}\right) \in B \times B$, any unit vector $\boldsymbol{n} \in \mathbb{R}^{d}$, and any $t>0$, the space average over the Riemann fan of the entropy solution $\boldsymbol{u}\left(\boldsymbol{n}, \boldsymbol{u}_{L}, \boldsymbol{u}_{R}\right)(x, t)$ remains in $B$ for all $t>0$.

We are also going to make use of the notion of invariant domain for an approximation process. Let $\boldsymbol{X}_{h} \subset L^{1}\left(\mathbb{R}^{d} ; \mathbb{R}^{m}\right)$ be a finite-dimensional approximation space and let $S_{h}: \boldsymbol{X}_{h} \ni \boldsymbol{u}_{h} \longmapsto S_{h}\left(\boldsymbol{u}_{h}\right) \in \boldsymbol{X}_{h}$ be a mapping over $\boldsymbol{X}_{h}$. (Think of $S_{h}$ as being a one-time-step approximation of (2.1).) Henceforth we abuse the language by saying that a member of $\boldsymbol{X}_{h}$, say $\boldsymbol{u}_{h}$, is in the set $B \subset \mathbb{R}^{m}$ when actually we mean that $\left\{\boldsymbol{u}_{h}(\boldsymbol{x}) \mid \boldsymbol{x} \in \mathbb{R}^{d}\right\} \subset B$.

Definition 2.2 (Invariant domain). A convex invariant set $B \subset \mathcal{A} \subset \mathbb{R}^{m}$ is said to be an invariant domain for the mapping $S_{h}$ if and only if for any state $\boldsymbol{u}_{h}$ in $B$, the state $S_{h}\left(\boldsymbol{u}_{h}\right)$ is also in $B$. 
It is known that the set

$$
A_{s^{\min }}:=\left\{(\rho, \boldsymbol{m}, E) \mid \rho>0, e>0, s \geq s^{\min }\right\}
$$

is invariant for the Euler system for any $s^{\min } \in \mathbb{R}$. It is also established in Serre [38, Thm. 8.2.2] that the set $A_{s^{\min }}$ is convex, and it is shown in Frid [7, Thm. 7 and 8] that it is an invariant domain for the Lax-Friedrichs scheme. The finite element method introduced in Guermond and Popov [11] also satisfies this invariant domain property; this finite element construction is recalled in $§ 3.1$. It is generally admitted in the literature that physical solutions to (2.1) should satisfy entropy inequalities. More specifically, let $f: \mathbb{R} \rightarrow \mathbb{R}$ be twice differentiable and be such that

$$
f^{\prime}(s)>0, \quad f^{\prime}(s) c_{p}^{-1}-f^{\prime \prime}(s)>0, \quad \forall(\rho, e) \in \mathbb{R}_{+}^{2},
$$

where $c_{p}(\rho, e)=T \partial_{T} s(p, T)$ is the specific heat at constant pressure. It is shown in Harten et al. [19, Thm. 2.1] that $\rho f(s)$ is strictly concave with respect to the conservative variables if and only if (2.5) holds, i.e., $\mathcal{A} \ni \boldsymbol{u} \mapsto \rho f(\Phi(\boldsymbol{u}))$ is convex. Note that the so-called physical entropy $S(\boldsymbol{u}):=\rho s$ is obtained by setting $f(s)=s$. We say that a weak solution to (2.1) is an entropy solution if it satisfies the following inequality in the weak sense for every generalized entropy:

$$
\partial_{t}(\rho f(s))+\nabla \cdot(\boldsymbol{m} f(s)) \geq 0 .
$$

In particular it is known (at least for $\gamma$-law equation of state) that the entropy solution to the Riemann problem (2.3) satisfy (2.6). Both the Lax-Friedrichs scheme and the finite element method introduced in [11] satisfy a discrete version of (2.6) for every generalized entropy.

The objective of the present work is to construct an explicit, second-order continuous finite element method that is consistent with (2.6) and for which $A_{s^{\min }}$ is an invariant domain.

2.3. Finite element setting. We are going to approximate the solution of (2.1) with continuous Lagrange finite elements. We introduce for this purpose a shaperegular sequence of matching meshes $\left(\mathcal{T}_{h}\right)_{h>0}$ and assume that the elements in each mesh are generated from a small collection of reference elements denoted $\widehat{K}_{1}, \ldots, \widehat{K}_{\varpi}$. In two space dimensions for instance, the mesh $\mathcal{T}_{h}$ could be composed of a combination of parallelograms and triangles (i.e., $\varpi=2$ ). In three space dimensions, $\mathcal{T}_{h}$ could also be composed of a combination of triangular prisms, parallelepipeds, and tetrahedra ( $\varpi=3$ ). Given $K \in \mathcal{T}_{h}$, the geometric transformation mapping $\widehat{K}_{r}$ to $K \in \mathcal{T}_{h}$ is denoted $T_{K}: \widehat{K}_{r} \longrightarrow K$. We are going to construct the approximation space by using some reference Lagrange finite elements $\left\{\left(\widehat{K}_{r}, \widehat{P}_{r}, \widehat{\Sigma}_{r}\right)\right\}_{1 \leq r \leq \varpi}$, where the objects $\left(\widehat{K}_{r}, \widehat{P}_{r}, \widehat{\Sigma}_{r}\right)$ are Ciarlet triples (we omit the index $r \in\{1: \varpi\}$ in the rest of the paper to simplify the notation). Given a reference Lagrange element $(\widehat{K}, \widehat{P}, \widehat{\Sigma})$, we denote by $\left\{\widehat{\boldsymbol{x}}_{l}\right\}_{l \in \mathcal{L}}$ the reference Lagrange nodes and by $\left\{\widehat{\theta}_{l}\right\}_{l \in \mathcal{L}}$ the reference shape functions, i.e., $\operatorname{card}(\mathcal{L})=\operatorname{dim}(\widehat{P})=: n_{\text {sh }}$ (note that the index $r$ has been omitted). $\widehat{P}$ is the reference approximation space (usually a scalar-valued polynomial space) and $\widehat{\Sigma}$ is the set of the Lagrange degrees of freedom. Let $\mathbb{P}_{l, d}$ be the vector space composed of the $d$-variate polynomials of degree at most $l$. We henceforth assume that there is $k \geq 1$ such that $\mathbb{P}_{k, d} \subset \widehat{P}$. The reference degrees of freedom $\left\{\widehat{\sigma}_{l}\right\}_{l \in \mathcal{L}}$ are such that $\widehat{\sigma}_{l}(\widehat{p})=\widehat{p}\left(\widehat{\boldsymbol{x}}_{l}\right)$ for all $l \in \mathcal{L}$ and all $\widehat{p} \in \widehat{P}$. By definition $\widehat{\theta}_{l}\left(\widehat{\boldsymbol{x}}_{l^{\prime}}\right)=\delta_{l l^{\prime}}$, for all $l . l^{\prime} \in \mathcal{L}$, which is turn implies the partition of unity property: $\sum_{l \in \mathcal{L}} \widehat{\theta}_{l}(\widehat{\boldsymbol{x}})=1$, for all $\widehat{\boldsymbol{x}} \in \widehat{K}$. 
Setting $\left\{\widehat{w}_{l}:=\int_{\widehat{K}} \theta_{l} \mathrm{~d} x\right\}_{l \in \mathcal{L}}$, the following quadrature is $(k+1)$-th order accurate since it is exact for all $\widehat{p} \in \widehat{P}: \int_{\widehat{K}} \widehat{p}(\widehat{\boldsymbol{x}}) \mathrm{d} \widehat{\boldsymbol{x}}=\sum_{l \in \mathcal{L}} \widehat{p}\left(\widehat{\boldsymbol{x}}_{l}\right) \widehat{w}_{l}$. We henceforth assume that

$$
\int_{\widehat{K}} \widehat{\theta}_{l} \mathrm{~d} \widehat{\boldsymbol{x}}=: \widehat{w}_{l}>0, \quad l \in \mathcal{L}
$$

There are numerous reference finite elements satisfying (2.7). For instance all the elements based on $\mathbb{Q}_{k, d}$ polynomials using tensor product of Gauss-Lobatto points on quadrangles or hexahedra satisfy (2.7). The question is slightly more delicate for $\mathbb{P}_{k, d}$ polynomials on simplices, but one can use Fekete points (see Taylor et al. [40]) or various variations thereof for $k \geq 3$ (see Hesthaven [20], Warburton [43]). Note that the standard $\mathbb{P}_{1, d}$ Lagrange element satisfies (2.7) but $\mathbb{P}_{2, d}$ does not for $d \geq 2$.

We now introduce approximation spaces constructed as usual by using the pullback by the geometric transformation. More precisely we define the following scalarvalued and vector-valued finite element spaces:

$$
P\left(\mathcal{T}_{h}\right)=\left\{v \in \mathcal{C}^{0}(D ; \mathbb{R}) \mid v_{\mid K} \circ T_{K} \in \widehat{P}, \forall K \in \mathcal{T}_{h}\right\}, \quad \boldsymbol{P}\left(\mathcal{T}_{h}\right)=\left[P\left(\mathcal{T}_{h}\right)\right]^{d+2} .
$$

The global shape functions in $P\left(\mathcal{T}_{h}\right)$, which we recall form a basis of $P\left(\mathcal{T}_{h}\right)$, are denoted by $\left\{\varphi_{i}\right\}_{i \in \mathcal{I}}$, i.e., $\operatorname{card}(\mathcal{I})=\operatorname{dim}\left(P\left(\mathcal{T}_{h}\right)\right)$. The global Lagrange nodes are denoted $\left\{\boldsymbol{x}_{i}\right\}_{i \in \mathcal{I}}$. Upon introducing the connectivity array $\mathrm{j}: \mathcal{T}_{h} \times \mathcal{L} \longrightarrow \mathcal{I}$, we have $\varphi_{\mathrm{j}(l, K)}(\boldsymbol{x})=\widehat{\theta}_{l}\left(\left(T_{K}\right)^{-1}(\boldsymbol{x})\right)$, and $\boldsymbol{x}_{\mathrm{j}(K, l)}=T_{K}\left(\widehat{\boldsymbol{x}}_{l}\right)$, for all $l \in \mathcal{L}$ and all $K \in \mathcal{T}_{h}$. This implies that $\varphi_{i}\left(\boldsymbol{x}_{j}\right)=\delta_{i j}$. The local partition of unity property implies that

$$
\sum_{i \in \mathcal{I}} \varphi_{i}(\boldsymbol{x})=1, \quad \forall \boldsymbol{x} \in D
$$

Upon defining $m_{i}:=\int_{D} \varphi_{i}(\boldsymbol{x}) \mathrm{d} \boldsymbol{x}$, the above definitions imply that the following quadrature is $(k+1)$-th order accurate $\int_{D} v(\boldsymbol{x}) \mathrm{d} \boldsymbol{x}=\sum_{i \in \mathcal{I}} m_{i} v\left(\boldsymbol{x}_{i}\right)$ since it is exact for all $v \in P\left(\mathcal{T}_{h}\right)$. The matrix with entries $\int_{D} \varphi_{i}(\boldsymbol{x}) \varphi_{j}(\boldsymbol{x}) \mathrm{d} \boldsymbol{x}$ is called the consistent mass matrix and denoted by $\mathcal{M} \in \mathbb{R}^{I \times I}$. Using the above quadrature and the property $\varphi_{i}\left(\boldsymbol{x}_{j}\right)=\delta_{i j}$, the integral $\int_{D} \varphi_{i}(\boldsymbol{x}) \varphi_{j}(\boldsymbol{x}) \mathrm{d} \boldsymbol{x}$ can be approximated by $m_{i}$. Note that (2.9) implies that $\sum_{j \in \mathcal{I}} m_{i j}=m_{i}$. We henceforth denote by $\mathcal{M}^{L}$ the diagonal matrix with entries $\left(m_{i}\right)_{i \in \mathcal{I}}$ and refer to $\mathcal{M}^{L}$ as the lumped mass matrix. Note that (2.7) implies that $m_{i}>0$ for all $i \in \mathcal{I}$.

We denote by $D_{i}$ the support of $\varphi_{i}$. Let $E$ be a union of cells in $\mathcal{T}_{h}$; we define $\mathcal{I}(E)$ to be the set containing the indices of all the shape functions whose support on $E$ is of nonzero measure. The sets $\mathcal{I}(K)$ and $\mathcal{I}\left(D_{i}\right)$ will be invoked regularly; note in particular that the partition of unity can be rewritten $\sum_{i \in \mathcal{I}(K)} \varphi_{i}(\boldsymbol{x})=1$ for all $\boldsymbol{x} \in K$.

Upon denoting by $\|\cdot\|_{\ell^{2}}$ the Euclidean norm in $\mathbb{R}^{d}$, we introduce the following two quantities which will play an important in the rest of paper:

$$
\boldsymbol{c}_{i j}:=\int_{D} \varphi_{i} \nabla \varphi_{j} \mathrm{~d} x, \quad \boldsymbol{n}_{i j}:=\frac{\boldsymbol{c}_{i j}}{\left\|\boldsymbol{c}_{i j}\right\|_{\ell^{2}}} \quad i, j \in \mathcal{I} .
$$

Note that (2.9) implies $\sum_{j \in\{1: I\}} \boldsymbol{c}_{i j}=\mathbf{0}$. Furthermore, if either $\varphi_{i}$ or $\varphi_{j}$ is zero on $\partial D$, then $\boldsymbol{c}_{i j}=-\boldsymbol{c}_{j i}$. In particular we have $\sum_{i \in\{1: I\}} \boldsymbol{c}_{i j}=\mathbf{0}$ if $\varphi_{j}$ is zero on $\partial D$. This property will be used to establish conservation. 
3. Time and space approximation. We describe in this section the first-order technique and the higher-order technique that will be used to construct the secondorder invariant domain preserving and entropy compliant method which is the object of the present paper. The discussion is restricted to the forward Euler time stepping since higher-order accuracy in time is trivially achieved by using Strong Stability Preserving Runge-Kutta time stepping. All the numerical tests reported in $\S 5$ are done with the SSP RK(3,3) method (three stages, third-order), see Shu and Osher [39, Eq. (2.18)] and Kraaijevanger [26, Thm. 9.4].

3.1. Low-order approximation (GMS-GV1). The low-order method that we are going to use is fully described in [11] and is henceforth referred to as GMSGV1 for Guaranteed Maximum Speed method with first-order Graph Viscosity. Let $\boldsymbol{u}_{h}^{0}=\sum_{i=1}^{I} \mathbf{U}_{i}^{0} \varphi_{i} \in \boldsymbol{P}\left(\mathcal{T}_{h}\right)$ be a reasonable approximation of the initial data $\boldsymbol{u}_{0}$. Let $\tau$ be the time step, $t_{n}$ be the current time, and let us set $t_{n+1}=t_{n}+\tau$ for some $n \in \mathbb{N}$. Letting $\boldsymbol{u}_{h}^{n}=\sum_{i \in \mathcal{I}}^{I} \mathbf{U}_{i}^{n} \varphi_{i}$ be the space approximation of $\boldsymbol{u}$ at time $t_{n}$, we estimate the low-order approximation $\boldsymbol{u}_{h}^{L, n+1}=\sum_{i \in \mathcal{I}} \mathbf{U}_{i}^{L, n+1} \varphi_{i}$ by setting

$$
\frac{m_{i}}{\tau}\left(\mathbf{U}_{i}^{L, n+1}-\mathbf{U}_{i}^{n}\right)+\sum_{j \in \mathcal{I}\left(D_{i}\right)} \boldsymbol{f}\left(\mathbf{U}_{j}^{n}\right) \cdot \boldsymbol{c}_{i j}-d_{i j}^{L, n}\left(\mathbf{U}_{j}^{n}-\mathbf{U}_{i}^{n}\right)=0 .
$$

The graph viscosity coefficients $d_{i j}^{L, n}$ are defined for all $i \neq j \in \mathcal{I}$ by

$$
d_{i j}^{L, n}:=\max \left(\lambda_{\max }\left(\boldsymbol{n}_{i j}, \mathbf{U}_{i}^{n}, \mathbf{U}_{j}^{n}\right)\left\|\boldsymbol{c}_{i j}\right\|_{\ell^{2}}, \lambda_{\max }\left(\boldsymbol{n}_{j i}, \mathbf{U}_{j}^{n}, \mathbf{U}_{i}^{n}\right)\left\|\boldsymbol{c}_{j i}\right\|_{\ell^{2}}\right),
$$

where $\lambda_{\max }\left(\boldsymbol{n}, \mathbf{U}_{L}, \mathbf{U}_{R}\right)$ is the maximum wave speed in the Riemann problem:

$$
\partial_{t} \boldsymbol{w}+\partial_{x} \boldsymbol{f}^{1 \mathrm{D}}(\boldsymbol{w})=0
$$

with data $\boldsymbol{w}_{L}:=\left(\rho_{L}, m_{L}, \mathcal{E}_{L}\right)^{\top}, \boldsymbol{w}_{R}:=\left(\rho_{R}, m_{R}, \mathcal{E}_{R}\right)^{\top}$, where $m:=\boldsymbol{m} \cdot \boldsymbol{n}, v:=m / \rho$, $\boldsymbol{m}^{\perp}:=\boldsymbol{m}-(\boldsymbol{m} \cdot \boldsymbol{n}) \boldsymbol{n}, \mathcal{E}:=E-\frac{1}{2} \frac{\left\|\boldsymbol{m}^{\perp}\right\|_{\ell 2}^{2}}{\rho}$, and flux $\boldsymbol{f}^{1 \mathrm{D}}(\boldsymbol{w}):=(m, v m+p, v(\mathcal{E}+p))^{\top}$. A guaranteed upper bound on $\lambda_{\max }\left(\boldsymbol{n}, \mathbf{U}_{L}, \mathbf{U}_{R}\right)$ is given in [11, Rem. 2.8] and Guermond and Popov [12, Appendix C] for the covolume equation of state $p(1-b \rho)=(\gamma-1) e \rho$, with $b \geq 0$; the case $b=0$ corresponds to an ideal gas. Let $v_{L}:=\boldsymbol{m}_{L} \cdot \boldsymbol{n} / \rho_{L}$, $v_{R}:=\boldsymbol{m}_{R} \cdot \boldsymbol{n} / \rho_{R}$ be the left and right speed and let $c_{L}, c_{R}$ the left and right speed of sound. We recall in passing that the widely used estimate $\max \left(\left|v_{L}\right|+c_{L},\left|v_{R}\right|+c_{R}\right)$ is not a a guaranteed upper bound of $\lambda_{\max }\left(\boldsymbol{n}, \mathbf{U}_{L}, \mathbf{U}_{R}\right)$. For the reader's convenience, we now recall the upper bound proposed in Guermond and Popov [12]. The definition of the maximum speed $\lambda_{\max }\left(\boldsymbol{n}, \mathbf{U}_{L}, \mathbf{U}_{R}\right)=\lambda_{\max }\left(\boldsymbol{w}_{L}, \boldsymbol{w}_{R}\right)$ is as follows:

$$
\lambda_{\max }\left(\boldsymbol{w}_{L}, \boldsymbol{w}_{R}\right)=\max \left(\left(\lambda_{1}^{-}\right)_{-},\left(\lambda_{3}^{+}\right)_{+}\right),
$$

where $\lambda_{1}^{-}$and $\lambda_{3}^{+}$are the two extreme wave speeds enclosing the Riemann fan of the one-dimensional problem (3.3); these two extreme wave speeds are given by

$$
\begin{aligned}
& \lambda_{1}^{-}\left(p^{*}\right)=v_{L}-c_{L}\left(1+\frac{\gamma+1}{2 \gamma}\left(\frac{p^{*}-p_{L}}{p_{L}}\right)_{+}\right)^{\frac{1}{2}} \\
& \lambda_{3}^{+}\left(p^{*}\right)=v_{R}+c_{R}\left(1+\frac{\gamma+1}{2 \gamma}\left(\frac{p^{*}-p_{R}}{p_{R}}\right)_{+}\right)^{\frac{1}{2}}
\end{aligned}
$$


where the intermediate pressure $p^{*}$ is obtained by solving a nonlinear problem. Here we use the notation $z_{+}:=\max (0, z)$ and we recall that the local sound speed for the covolume gas is $c=\sqrt{\frac{\gamma p}{\rho(1-b \rho)}}$. Note that the exact value of value of $\lambda_{\max }\left(\boldsymbol{w}_{L}, \boldsymbol{w}_{R}\right)\left(p^{*}\right)$ is a monotone increasing function of $p^{*}$. Therefore, instead of computing the exact pressure $p^{*}$ which requires an iterative process, one can use an explicit upper bound $\tilde{p}^{*} \geq p^{*}$. Then, a guaranteed upper bound on $\lambda_{\max }\left(\boldsymbol{w}_{L}, \boldsymbol{w}_{R}\right)\left(p^{*}\right)$ is $\lambda_{\max }\left(\boldsymbol{w}_{L}, \boldsymbol{w}_{R}\right)\left(\tilde{p}^{*}\right)$. One such upper bound valid for $1<\gamma \leq \frac{5}{3}$ is established in Guermond and Popov $[12$, Lem. 4.3$]$ and the value of $\tilde{p}^{*}$ in question is given by the so-called two-rarefaction approximation:

$$
\tilde{p}^{*}=\left(\frac{c_{L}\left(1-b \rho_{L}\right)+c_{R}\left(1-b \rho_{R}\right)-\frac{\gamma-1}{2}\left(v_{R}-v_{L}\right)}{c_{L}\left(1-b \rho_{L}\right) p_{L}^{-\frac{\gamma-1}{2 \gamma}}+c_{R}\left(1-b \rho_{R}\right) p_{R}^{-\frac{\gamma-1}{2 \gamma}}}\right)^{\frac{2 \gamma}{\gamma-1}} .
$$

Remark 3.1 (Graph Viscosity). Note that the expression $-d_{i j}^{L, n}\left(\mathbf{U}_{j}^{n}-\mathbf{U}_{i}^{n}\right)$ in (3.1) is called weighted Graph Laplacian in graph theory. We call the weights $d_{i j}^{L, n}$ Graph Viscosity (or artificial viscosity).

Remark 3.2 (Other discretizations). Note that the expression (3.1) that is used to compute the update $\mathbf{U}_{i}^{n+1}$ is quite generic; many other discretizations of the Euler equations can be put in this abstract form. The notion of continuous finite element only intervenes in the definition of the vectors $\boldsymbol{c}_{i j}$, the index set $\mathcal{I}\left(D_{i}\right)$, and the lumped mass matrix coefficients $m_{i}$. Other discretizations lead to other forms for $\boldsymbol{c}_{i j}, \mathcal{I}\left(D_{i}\right)$, and $m_{i}$. Almost everything that is said in the rest of the paper can be applied to these discretizations as well.

3.2. The intermediate limiting states. We now deduce from (3.1) intermediate local states that will be useful to limit the yet to be defined high-order solution. Using that $\sum_{j \in \mathcal{I}\left(D_{i}\right)} \boldsymbol{c}_{i j}=0$, we rewrite (3.1) as follows:

$$
\frac{m_{i}}{\tau} \mathbf{U}_{i}^{L, n+1}=\mathbf{U}_{i}^{n}\left(\frac{m_{i}}{\tau}-\sum_{i \neq j \in \mathcal{I}\left(D_{i}\right)} 2 d_{i j}^{L, n}\right)+\sum_{i \neq j \in \mathcal{I}\left(D_{i}\right)}\left(\boldsymbol{f}\left(\mathbf{U}_{i}^{n}\right)-\boldsymbol{f}\left(\mathbf{U}_{j}^{n}\right)\right) \cdot \boldsymbol{c}_{i j}+d_{i j}^{L, n}\left(\mathbf{U}_{j}^{n}+\mathbf{U}_{i}^{n}\right) .
$$

Then, upon introducing the quantities

$$
\overline{\mathbf{U}}_{i j}^{n+1}:=\frac{1}{2}\left(\mathbf{U}_{i}^{n}+\mathbf{U}_{j}^{n}\right)-\left(\boldsymbol{f}\left(\mathbf{U}_{j}^{n}\right)-\boldsymbol{f}\left(\mathbf{U}_{i}^{n}\right)\right) \cdot \frac{\boldsymbol{c}_{i j}}{2 d_{i j}^{L, n}},
$$

with the the convention $\overline{\mathbf{U}}_{i i}^{n+1}=\mathbf{U}_{i}^{n}$, and the notation $\overline{\mathbf{U}}_{i j}^{n+1}=\left(\bar{\rho}_{i j}^{n+1}, \overline{\boldsymbol{m}}_{i j}^{n+1}, \bar{E}_{i j}^{n+1}\right)^{\top}$ the low-order update $\mathbf{U}_{i}^{L, n+1}$, can be represented as a convex combination as follows:

$$
\mathbf{U}_{i}^{L, n+1}=\left(1-\sum_{i \neq j \in \mathcal{I}\left(D_{i}\right)} \frac{2 \tau d_{i j}^{L, n}}{m_{i}}\right) \overline{\mathbf{U}}_{i i}^{n}+\sum_{i \neq j \in \mathcal{I}\left(D_{i}\right)}\left(\frac{2 \tau d_{i j}^{L, n}}{m_{i}}\right) \overline{\mathbf{U}}_{i j}^{n+1},
$$

under the appropriate CFL condition. Lemma 2.1 from [12], inspired by Perthame and Shu $[36, \S 5]$ and Nessyahu and Tadmor [34, Eq. (2.7)], is that the intermediate state $\overline{\mathbf{U}}_{i j}^{n+1}$ is a space average of the Riemann solution $\boldsymbol{u}\left(\boldsymbol{n}_{i j}, \mathbf{U}_{i}^{n}, \mathbf{U}_{j}^{n}\right)$; that is to say, $\overline{\mathbf{U}}_{i j}^{n+1}=$ $\int_{-\frac{1}{2}}^{\frac{1}{2}} \boldsymbol{u}\left(\boldsymbol{n}_{i j}, \mathbf{U}_{i}^{n}, \mathbf{U}_{j}^{n}\right)(x, t) \mathrm{d} x$ with $t:=\left\|\boldsymbol{c}_{i j}\right\|_{\ell^{2}} /\left(2 d_{i j}^{L, n}\right)$ provided $t \lambda_{\max }\left(\boldsymbol{n}_{i j}, \mathbf{U}_{i}^{n}, \mathbf{U}_{j}^{n}\right) \leq$ $\frac{1}{2}$. (Let us emphasize tat the time $t$ is related to the Riemann problem (3.3), this time 
has noting to do with that of the PDE (2.1).) Note that the definition of the loworder graph viscosity $(3.2)$ does imply that $t \lambda_{\max }\left(\boldsymbol{n}_{i j}, \mathbf{U}_{i}^{n}, \mathbf{U}_{j}^{n}\right) \leq \frac{1}{2}$. An immediate consequence of this structure is that $\overline{\mathbf{U}}_{i j}^{n+1}$ has positive density, positive internal energy and satisfies the following minimum principle on the specific entropy: $\Phi\left(\overline{\mathbf{U}}_{i j}^{n+1}\right) \geq$ $\min \left(\Phi\left(\mathbf{U}_{i}^{n}\right), \Phi\left(\mathbf{U}_{j}^{n}\right)\right)$. Upon denoting $d_{i i}^{L, n}:=-\sum_{i \neq j \in \mathcal{I}\left(D_{i}\right)} d_{i j}^{L, n}$ for brevity, another consequence of the above observation is the following result.

THEOREM 3.3 (Local invariance/entropy inequality). Let $i \in \mathcal{I}$. Assume (2.7) and $1+2 \tau \frac{d_{i i}^{L, n}}{m_{i}} \geq 0$. (i) Let $s_{i}^{\min }=\min _{j \in \mathcal{I}\left(D_{i}\right)} \Phi\left(\mathbf{U}_{j}^{n}\right)$, then $\mathbf{U}_{i}^{L, n+1} \in A_{s_{i}^{\min }}$. (ii) Let $(\eta:=\rho f(\Phi), \boldsymbol{q}:=\boldsymbol{m} f(\Phi))$ be a generalized entropy pair for (2.1). Then the following local entropy inequality holds:

$$
\begin{aligned}
\frac{m_{i}}{\tau}\left(\eta\left(\mathbf{U}_{i}^{L, n+1}\right)-\eta\left(\mathbf{U}_{i}^{n}\right)\right)+\int_{D} \nabla \cdot\left(\sum_{j \in \mathcal{I}\left(D_{i}\right)} \boldsymbol{q}\left(\mathbf{U}_{j}^{n}\right) \varphi_{j}\right) \varphi_{i} \mathrm{~d} \boldsymbol{x} & \\
& -\sum_{j \in \mathcal{I}\left(D_{i}\right)} d_{i j}^{L, n}\left(\eta\left(\mathbf{U}_{j}^{n}\right)-\eta\left(\mathbf{U}_{j}^{n}\right)\right) \geq 0 .
\end{aligned}
$$

A practical interpretation of item (i) is that the low-order solution $\mathbf{U}_{i}^{L, n+1}$ has positive density, positive internal energy and satisfies the local minimum principle on the specific entropy. Item (ii) shows that this solution is also entropy satisfying in some discrete sense. This result is proved in [11, Thm. 4.7] in a more general setting for any hyperbolic system with a convex entropy. Note that in mathematical papers the entropies are generally assumed to be convex whereas the physical generalized entropies $\rho f(\Phi)$ are concave (this is just a matter of sign convention). Hence, Theorem 4.7 in [11] can be applied with $-\rho f(\Phi)$.

3.3. Smoothness-based approximation. In this section and the following one we introduce high-order approximation techniques that will provide us with a provisional high-order solution $\mathbf{U}_{j}^{H, n+1}$. The method presented in this section is easy to implement but is inherently only second-order accurate in space.

We introduce a technique to reduce the graph viscosity that is based on a measure of the local smoothness of the solution in the spirit of the finite volume literature (see e.g., Jameson et al. [23, Eq. (12)] and see the second formula in the right column of page 1490 in Jameson [22]). Given a scalar-valued function $g$ and its finite element interpolant $g_{h}=\sum \mathrm{G}_{i} \varphi_{i}$, and denoting $\epsilon_{i}=\epsilon \max _{j \in \mathcal{I}\left(D_{i}\right)} \mathrm{G}_{j}$ where $\epsilon=10^{-\frac{16}{2}}$ in double precision arithmetic, we define the smoothness indicator

$$
\alpha_{i}\left(g_{h}\right):=\frac{\left|\sum_{j \in \mathcal{I}\left(S_{i}\right)} \beta_{i j}\left(\mathrm{G}_{j}-\mathrm{G}_{i}\right)\right|}{\max \left(\sum_{j \in \mathcal{I}\left(S_{i}\right)} \beta_{i j}\left|\mathrm{G}_{j}-\mathrm{G}_{i}\right|, \epsilon_{i}\right)},
$$

where the real numbers $\beta_{i j}$ are assumed to be positive. One can use the parameters $\beta_{i j}$ to make $\alpha_{i}=0$ if $g_{h}$ is linear on the support of the shape function $\varphi_{i}$, this property is called linearity-preserving (see Berger et al. [3] for a review on linearity-preserving limiters in the finite volume literature). Note that $\alpha_{i} \in[0,1]$ for all $i \in\{1: I\}$ and $\alpha_{i}=$ 1 if $\mathrm{G}_{i}$ is a local extrema of $g_{h}$. Moreover, if the coefficients $\beta_{i j}$ are defined so that $\alpha_{i}=$ 0 if $g_{h}$ is linear on $S_{i}$, then the numerator of (3.10) behaves like $h^{2}\left\|D^{2} g(\boldsymbol{\xi})\right\|_{\ell^{2}\left(\mathbb{R}^{d \times d}\right)}$ at some point $\boldsymbol{\xi}$, whereas the denominator behaves like $h\|\nabla g(\boldsymbol{\zeta})\|_{\ell^{2}\left(\mathbb{R}^{d}\right)}$ at some point $\boldsymbol{\zeta}$. Therefore, we have $\alpha_{i} \approx h\left\|D^{2} g(\boldsymbol{\xi})\right\|_{\ell^{2}\left(\mathbb{R}^{d \times d}\right)} /\|\nabla g(\boldsymbol{\zeta})\|_{\ell^{2}\left(\mathbb{R}^{d}\right)}$, that is to say $\alpha_{i}$ is of 
order $h$ in the regions where $g$ is smooth and does not have a local extremum. In the computations reported at the end of the paper we have taken $\beta_{i j}=1$.

One choice for $g$ that we consider in some numerical tests reported at the end of the paper consists using $g(\boldsymbol{u})=\rho \Phi(\boldsymbol{u})=S(\boldsymbol{u})$, which is the mathematical entropy (up to a sign). Other options consists of using generalized entropies of the Euler equations, $g(\boldsymbol{u})=\rho f(\Phi(\boldsymbol{u}))$. In particular, taking $f(s)=1$, gives $g(\boldsymbol{u})=\rho$, which is an extreme case of generalized entropy; it is extreme in the sense that $-g(\boldsymbol{u})$ is convex but not strictly convex. Note in passing that it is shown in Guermond and Popov [14] that using $g(\boldsymbol{u})=\rho$ guarantees positivity of the density. Another option, which is somewhat similar to Jameson et al. [23, Eq. (12)], Jameson [22, p. 1490], consists of taking $g(\boldsymbol{u})=p$. Note however that it might be better to take $p^{\frac{1}{\gamma}}$ to be entropy consistent, since $p^{\frac{1}{\gamma}}$ is an extreme generalized entropy for polytropic gas as shown in Harten [17, Eq. (2.10a)]. Let us emphasize that strict convexity of the entropy is not needed for the purpose of the present paper.

Let $\psi \in C^{0,1}([0,1] ;[0,1])$ be any positive function such that $\psi(1)=1$. The high-order smoothness-based graph viscosity is defined by setting

$$
d_{i j}^{H, n}:=d_{i j}^{L, n} \max \left(\psi\left(\alpha_{i}^{n}\right), \psi\left(\alpha_{j}^{n}\right)\right), \quad d_{i i}^{H, n}:=-\sum_{i \neq j \in \mathcal{I}\left(D_{i}\right)} d_{i j}^{H, n},
$$

where $\alpha_{i}^{n}:=\alpha_{i}\left(g_{h}^{n}\right)$. A typical choice for $\psi$ consists of setting $\psi(\alpha)=\alpha^{2}$. Then the provisional high-order approximation is computed as follows:

$$
\sum_{j \in \mathcal{I}\left(D_{i}\right)} \frac{m_{i j}}{\tau}\left(\mathbf{U}_{j}^{H, n+1}-\mathbf{U}_{j}^{n}\right)+\sum_{j \in \mathcal{I}\left(D_{i}\right)} \boldsymbol{f}\left(\mathbf{U}_{j}^{n}\right) \cdot \boldsymbol{c}_{i j}-d_{i j}^{H, n}\left(\mathbf{U}_{j}^{n}-\mathbf{U}_{i}^{n}\right)=0 .
$$

Note that we use the consistent mass matrix to reduce dispersion error since it is known that the use of the consistent mass matrix corrects the dominating dispersion error (at least for piecewise linear approximation), see Christon et al. [5], Gresho et al. [9], Guermond and Pasquetti [10], Thompson [41]. The beneficial effects of the consistent mass matrix are particularly visible when solving problems with nonsmooth solutions, see e.g., [10, Fig. 5.5].

3.4. Entropy viscosity commutator. We now introduce a method that is formally high-order for any polynomial degree, contrary the one introduced in $§ 3.3$. Our objective is to construct a high-order method that is entropy consistent and close to be invariant domain preserving. We do not want to rely on the yet to be explained limiting process to enforce entropy consistency. We refer the reader to Lemma 3.2, Lemma 4.4 and $\S 6.1$ in Guermond and Popov [13] and Guermond and Popov [11, §5.1] for counter-examples of methods that are invariant domain preserving but entropy violating. The heuristics we have in mind is that limitation should be understood as a light polishing applied to a method that is already entropy consistent and almost invariant domain preserving. Following an idea introduced in Guermond et al. [15, 16], we construct a high-order graph viscosity that is entropy consistent by estimating a non-dimensional entropy residual. However, contrary to the techniques introduced in $[15,16]$, we do not want the time discretization to interfere with the estimation of the residual, and we now propose a slightly different approach. Given the current approximation $\boldsymbol{u}_{h}^{n}$, we estimate the next inviscid approximation by setting $\mathbf{U}_{i}^{G, n+1}:=\mathbf{U}_{i}^{n}-\frac{\tau}{m_{i}} \sum_{j \in \mathcal{I}\left(D_{i}\right)} \boldsymbol{f}\left(\mathbf{U}_{j}^{n}\right) \boldsymbol{c}_{i j}$. Essentially $\mathbf{U}_{i}^{G, n+1}$ is the Galerkin approximation of $\boldsymbol{u}\left(t^{n+1}\right)$. Let $(\eta(\boldsymbol{v}), \boldsymbol{F}(\boldsymbol{v}))$ be an entropy pair for (2.1). We estimate the entropy residual for the degree of freedom $i$ by computing 
$\frac{m_{i}}{\tau}\left(\mathbf{U}_{i}^{G, n+1}-\mathbf{U}_{i}^{n}\right) \cdot \eta^{\prime}\left(\mathbf{U}_{i}^{n}\right)+\sum_{j \in \mathcal{I}\left(D_{i}\right)} \boldsymbol{F}\left(\mathbf{U}_{j}^{n}\right) \boldsymbol{c}_{i j}$. But using the definition of $\mathbf{U}_{i}^{G, n+1}$, this is equivalent to computing $\sum_{j \in \mathcal{I}\left(D_{i}\right)}\left(\boldsymbol{F}\left(\mathbf{U}_{j}^{n}\right)-\eta^{\prime}\left(\mathbf{U}_{i}^{n}\right)^{\top} \boldsymbol{f}\left(\mathbf{U}_{j}^{n}\right)\right) \boldsymbol{c}_{i j}$. Then, upon set$\operatorname{ting} \eta_{i}^{\min }:=\min _{j \in \mathcal{I}\left(D_{i}\right)} \eta\left(\mathbf{U}_{j}^{n}\right), \eta_{i}^{\max }:=\max _{j \in \mathcal{I}\left(D_{i}\right)} \eta\left(\mathbf{U}_{j}^{n}\right), \epsilon_{i}:=\epsilon \max \left(\left|\eta_{i}^{\max }\right|,\left|\eta_{i}^{\min }\right|\right)$, we adopt the following definition

$$
R_{i}^{n}=\frac{1}{\max \left(\eta_{i}^{\max }-\eta_{i}^{\min }, \epsilon_{i}\right)} \sum_{j \in \mathcal{I}\left(D_{i}\right)}\left(\boldsymbol{F}\left(\mathbf{U}_{j}^{n}\right)-\eta^{\prime}\left(\mathbf{U}_{i}^{n}\right)^{\top} \boldsymbol{f}\left(\mathbf{U}_{j}^{n}\right)\right) \cdot \boldsymbol{c}_{i j}
$$

where $\epsilon$ a tiny number that avoids division by zero when the entropy is constant over $D_{i}$. In practice we take $\epsilon=10^{-\frac{16}{2}}$ in double precision arithmetic. Note that $R_{i}^{n}$ can be interpreted as a commutator. More specifically $R_{i}^{n}$ can be rewritten as follows $\frac{1}{\max \left(\eta_{i}^{\max }-\eta_{i}^{\min }, \epsilon_{i}\right)} \int_{D}\left(\nabla \cdot\left(\Pi_{h} \boldsymbol{F}\left(\boldsymbol{u}_{h}^{n}\right)\right)-\eta^{\prime}\left(\mathbf{U}_{i}^{n}\right)^{\top} \nabla \cdot\left(\Pi_{h}\left(\boldsymbol{f}\left(\boldsymbol{u}_{h}^{n}\right)\right)\right)\right) \varphi_{i}$ d $\boldsymbol{x}$ where, up to two slight abuses of notation, $\Pi_{h}: C^{0}(D) \rightarrow P\left(\mathcal{T}_{h}\right)$ is the Lagrange interpolation operator. Note in passing that $R_{i}^{n}=0$ in the hypothetical case that $\eta: \mathbb{R}^{d+2} \rightarrow \mathbb{R}$ is linear.

The high-order graph viscosity (or entropy viscosity, EV) is defined by

$$
d_{i j}^{H, n}=\min \left(d_{i j}^{L, n}, \max \left(\left|R_{i}^{n}\right|,\left|R_{j}^{n}\right|\right)\right), \quad d_{i i}^{H, n}:=-\sum_{i \neq j \in \mathcal{I}\left(D_{i}\right)} d_{i j}^{H, n},
$$

and the provisional high-order approximation is computed as follows:

$$
\sum_{j \in \mathcal{I}\left(D_{i}\right)} \frac{m_{i j}}{\tau}\left(\mathbf{U}_{j}^{H, n+1}-\mathbf{U}_{j}^{n}\right)+\sum_{j \in \mathcal{I}\left(D_{i}\right)} \boldsymbol{f}\left(\mathbf{U}_{j}^{n}\right) \cdot \boldsymbol{c}_{i j}-d_{i j}^{H, n}\left(\mathbf{U}_{j}^{n}-\mathbf{U}_{i}^{n}\right)=0 .
$$

Note again that we use the consistent mass matrix to reduce dispersion errors as explained in $\S 3.3$.

Remark 3.4 (Scaling of $R_{i}^{n}$ ). Let us now convince ourselves that $R_{i}^{n}$ is at least one order smaller (in term of mesh size) than $d_{i j}^{L, n}$. Let use denote $F_{\max }^{\prime \prime}$ and $f_{\max }^{\prime \prime}$ the maximum over the convex hull $\operatorname{conv}\left(\mathbf{U}_{j}^{n}\right)_{j \in \mathcal{I}\left(D_{i}\right)}$ of the matrix norm (say the norm induced by the Euclidean norm in $\mathbb{R}^{d+2}$ ) of the Hessians $D^{2} \boldsymbol{F}$ and $D^{2} f$. Then, denoting by $N_{i}^{n}$ the numerator in (3.13), and recalling that $D \boldsymbol{F}(\mathbf{U})=\eta^{\prime}(\mathbf{U})^{\top} D \boldsymbol{f}(\mathbf{U})$, we have

$$
\begin{aligned}
\left\|N_{i}^{n}\right\|_{\ell^{2}} & =\| \sum_{j \in \mathcal{I}\left(D_{i}\right)}\left(\boldsymbol{F}\left(\mathbf{U}_{j}^{n}\right)-\boldsymbol{F}\left(\mathbf{U}_{i}^{n}\right)-\eta^{\prime}\left(\mathbf{U}_{i}^{n}\right)^{\top}\left(\boldsymbol{f}\left(\mathbf{U}_{j}^{n}\right)-\boldsymbol{f}\left(\mathbf{U}_{i}^{n}\right) \cdot \boldsymbol{c}_{i j} \|_{\ell^{2}}\right.\right. \\
& \leq \frac{1}{2}\left(F_{\max }^{\prime \prime}+\eta^{\prime}\left(\mathbf{U}_{i}^{n}\right) f_{\max }^{\prime \prime}\right) \max _{j \in \mathcal{I}\left(D_{i}\right)}\left\|\boldsymbol{c}_{i j}\right\|_{\ell^{2}} \sum_{j \in \mathcal{I}\left(D_{i}\right)}\left\|\mathbf{U}_{j}^{n}-\mathbf{U}_{i}^{n}\right\|_{\ell^{2}}^{2} .
\end{aligned}
$$

Assuming that $\eta^{\prime}$ is not zero over $D_{i}$ and denoting by $\eta_{\min }^{\prime}$ the minimum of $\left\|\eta^{\prime}\right\|_{\ell^{2}}$ over $\operatorname{conv}\left(\mathbf{U}_{j}^{n}\right)_{j \in \mathcal{I}\left(D_{i}\right)}$, the quantity $\eta_{\min }^{\prime} \sum_{j \in \mathcal{I}\left(D_{i}\right)}\left\|\mathbf{U}_{j}^{n}-\mathbf{U}_{i}^{n}\right\|_{\ell^{2}}$ is a lower bound for the denominator in (3.13). The conclusion follows readily.

Remark 3.5 (Choice of high-order graph viscosity). One advantage we see in the entropy viscosity (3.14) over the smoothness-based viscosity (3.11) is that, in addition to being consistent for any polynomial degree, it is also consistent with at least one entropy inequality. That is to say the viscosity is large when the entropy production is large and it is small otherwise. In any case, we have observed that (3.14) always gives a scheme that is more robust than (3.11) albeit being slightly more oscillatory. We refer the reader to Guermond and Popov [13, §6.5] where this issue is discussed in detail. 
Remark 3.6 (Entropy). We have found in our numerical experiments that using $p^{\frac{1}{\gamma}}$ for polytropic gases is a very good choice to construct the entropy residual since it minimizes dissipation across contact discontinuities. Recall that $p^{\frac{1}{\gamma}}$ is indeed an entropy in the case of polytropic gases. All the numerical tests reported at the end of the paper are done with this entropy.

Remark 3.7 (Entropy ansatz). In realistic applications the equation of state is often tabulated and the entropy $\Phi(\mathbf{U})$ may be either unavailable or costly to estimate. We have found that the pressure may be used as an ansatz for the entropy; one can then replace (3.13) by $R_{i}^{n}=\frac{1}{\max \left(\mathrm{P}_{i}^{\max }-\mathrm{P}_{i}^{\min }, \epsilon_{i}\right)} \sum_{j \in \mathcal{I}\left(D_{i}\right)} \mathbf{V}_{i}^{n} \cdot \boldsymbol{c}_{i j}\left(\mathrm{P}_{j}^{n}-D \mathrm{P}_{i}^{n} \cdot \mathbf{U}_{j}^{n}\right)$ with $\epsilon_{i}=\epsilon \max \left(\left|\mathrm{P}_{i}^{\max }\right|,\left|\mathrm{P}_{i}^{\min }\right|\right)$.

4. Quasiconcavity-based limitation. In this section we discuss the bounds we want the numerical solution $\mathbf{U}_{i}^{n+1}$ to satisfy, and we develop a novel limiting technique that is convexity-based and does not invoke arguments like linearization, worst-case scenario estimates, a posteriori fixes, or auxiliary discontinuous spaces as often done in the literature. This technique takes its roots in Khobalatte and Perthame [25], Perthame and Qiu [35], and Perthame and Shu [36]. We also refer to Zhang and Shu $[47,48]$, Jiang and Liu [24] for extensions in the context of the Discontinuous Galerkin approximation.

4.1. Bounds and quasiconcavity. Since the high-order update $\mathbf{U}_{i}^{H, n+1}$ (using either (3.12) or (3.15)) is not guaranteed to be oscillation free and to preserve physical bounds, some form of limitation must applied. The question is now the following: what should be limited and how? Whichever representation is chosen for the dependent variable (conservative, primitive, or characteristic variables), the Euler equations are not known to satisfy any maximum or minimum principle, with the exception of the minimal principle on the specific entropy. Despite this fundamental negative result and with varying levels of success, a number of techniques have been proposed over the years in the finite element literature to enforce some kinds of discrete maximum principles (see for instance Boris and Book [4], Zalesak [45], Löhner et al. [32], Kuzmin and Möller [27], Zalesak [46], Lohmann and Kuzmin [31]). Some of these limiting techniques enforce properties that are not necessarily satisfied by the Euler equations, or in the best case scenario, satisfied by the first-order method of choice (usually a Lax-Friedrichs-like first-order scheme).

In the present paper, we take a different point of view. In addition to the local minimum principle on the specific entropy, the strategy that we propose consists of enforcing bounds that are naturally satisfied by the low-order solution. More precisely, let us set

$$
\begin{aligned}
\rho_{i}^{\min } & :=\min _{j \in \mathcal{I}\left(D_{i}\right)}\left(\bar{\rho}_{i j}^{n+1}, \rho_{j}^{n}\right), \quad \rho_{i}^{\max }:=\max _{j \in \mathcal{I}\left(D_{i}\right)}\left(\bar{\rho}_{i j}^{n+1}, \rho_{j}^{n}\right), \\
E_{i}^{\min } & :=\min _{j \in \mathcal{I}\left(D_{i}\right)}\left(\bar{E}_{i j}^{n+1}, E_{j}^{n}\right), \quad E_{i}^{\max }:=\max _{j \in \mathcal{I}\left(D_{i}\right)}\left(\bar{E}_{i j}^{n+1}, E_{j}^{n}\right), \\
s_{i}^{\min } & :=\min _{j \in \mathcal{I}\left(D_{i}\right)} \Phi\left(\mathbf{U}_{j}^{n}\right) .
\end{aligned}
$$

We have already established in $\S 3.2$ that $\rho_{i}^{\min } \leq \rho_{i}^{L, n+1} \leq \rho_{i}^{\max }, E_{i}^{\min } \leq E_{j}^{L, n+1} \leq$ $E_{i}^{\max }$ and $s_{i}^{\min } \leq \Phi\left(\mathbf{U}_{i}^{L, n+1}\right)$. In the next section we are going to modify the graph viscosity so that the resulting high-order update $\mathbf{U}_{i}^{n+1}$ satisfies $\rho_{i}^{\min } \leq \rho_{i}^{n+1} \leq \rho_{i}^{\max }$ and $s_{i}^{\min } \leq \Phi\left(\mathbf{U}_{i}^{n+1}\right)$ (and possibly $E_{i}^{\min } \leq E_{j}^{n+1} \leq E_{i}^{\max }$ if one wishes). 
In general, the equation for the specific entropy may not be explicitly available and therefore limiting the specific entropy may not be possible. An alternative strategy consists of limiting the internal energy $\rho e$. Using the Frechet derivative notation, a straightforward computation shows that $D^{2}(\rho e(\boldsymbol{u}))((\varrho, \boldsymbol{p}, a),(\varrho, \boldsymbol{p}, a))=-\frac{1}{\rho}\left(\frac{\varrho}{\rho} \boldsymbol{m}-\boldsymbol{p}\right)^{2}$ for all directions $(\varrho, \boldsymbol{p}, a)^{\top} \in \mathbb{R}^{d+2}$ and all points $\boldsymbol{u}=(\rho, \boldsymbol{m}, E)^{\top} \in \mathbb{R}^{d+2}$, thereby showing that the internal energy is concave with respect to the conservative variables irrespective of the equation of state. Hence, the concavity of $(\rho e)$ along with the convex combination (3.9) implies that the low-order solution $\mathbf{U}_{i}^{L, n+1}$ satisfies the following discrete minimum principle

$$
(\rho e)\left(\mathbf{U}_{i}^{L, n+1}\right) \geq \varepsilon_{i}^{\min }:=\min \left(\min _{j \in \mathcal{I}\left(D_{i}\right)}(\rho e)\left(\mathbf{U}_{j}^{n}\right), \min _{j \in \mathcal{I}\left(D_{i}\right)}(\rho e)\left(\overline{\mathbf{U}}_{i j}^{n+1}\right)\right) .
$$

In order to unify into one single framework all the bounds that we want to enforce, we are going to rely on the notion of quasiconcavity, which definition we now recall.

Definition 4.1 (Quasiconcavity). Given a convex set $\mathcal{A} \subset \mathbb{R}^{m}$, we say that a function $\Psi: \mathcal{A} \rightarrow \mathbb{R}$ is quasiconcave if every upper level set of $\Psi$ is convex; that is, the set $L_{\lambda}(\Psi):=\{\mathbf{U} \in \mathcal{A} \mid \Psi(\mathbf{U}) \geq \lambda\}$ is convex for any $\lambda \in \mathbb{R}$ in the range of $\Psi$.

Note in passing that concavity implies quasiconcavity. We are going to use the above definition in the following three settings: (i) $\mathcal{A}=\mathbb{R}^{d+2}$ and $\Psi(\mathbf{U})=\rho-\rho_{i}^{\min }$ or $\Psi(\mathbf{U})=\rho_{i}^{\max }-\rho$. Note that in both cases the upper level sets are half spaces (i.e., these sets are obviously convex); (ii) $\mathcal{A}=\{\mathbf{U}:=(\rho, \boldsymbol{m}, E) \mid \rho>0\}$ and $\Psi(\mathbf{U})=(\rho e)-\varepsilon_{i}^{\min }$. We have shown above that that $(\rho e)(\mathbf{U})$ is concave provided $\rho>0$ (the Hessian of $\rho e$ is nonpositive), then it follows that $\{\mathbf{U}:=(\rho, \boldsymbol{m}, E) \mid \rho>0, e>0\}$ is convex; (iii) $\mathcal{A}=\{\mathbf{U}:=(\rho, \boldsymbol{m}, E) \mid \rho>0, e>0\}, \Psi(\mathbf{U})=\Phi(\mathbf{U})-s_{i}^{\mathrm{min}}$. The quasiconvexity of $\Psi: \mathcal{A} \rightarrow \mathbb{R}$ is proved in Serre [38, Thm. 8.2.2].

Remark 4.2 (Concavity vs. quasiconcavity). Note that the two sets

$$
\{(\rho, \boldsymbol{m}, E) \mid \rho>0, e>0, s \geq r\}, \quad\{(\rho, \boldsymbol{m}, E) \mid \rho>0, \rho e>0, \rho(s-r) \geq 0\}
$$

are identical. In the first case, quasiconcavity is invoked to prove that the upper level sets are convex, whereas in the second case one just has to rely on concavity since the three functions $\rho, \rho e(\boldsymbol{u})$, and $\rho(\Phi(\boldsymbol{u})-r)$ are concave. It is easier to impose concave (or convex) constraints than quasiconcave ones. More precisely: in practice it is simpler to apply Newton's method on a concave function than on a quasiconcave function; in the first case Newton's method is guaranteed to converge independently of the initial guess, whereas it may not in the second case.

4.2. An abstract limiting scheme. Simple linear constraints such as $\bar{\rho}_{i}^{\text {min }} \leq$ $\rho_{i}^{n+1} \leq \bar{\rho}_{i}^{\max }$ and $\bar{E}_{i}^{\text {min }} \leq E_{j}^{n+1} \leq \bar{E}_{i}^{\max }$, can be easily enforced by using the Flux Transport Corrected paradigm of Zalesak [45] (see also Boris and Book [4]). However, to the best of our knowledge, the Zalezak's grouping methodology cannot be (easily) extended to handle general convex constraints like the minimum principle on the specific entropy without losing second-order accuracy. We introduce in this section a methodology that does exactly that.

We start as in the FCT methodology by estimating the difference $\mathbf{U}_{j}^{H, n+1}-$ $\mathbf{U}_{j}^{L, n+1}$. Subtracting (3.12) (or (3.15)) from (3.1) we obtain that the low-order and the provisional high-order solutions satisfy the following identity

$$
\sum_{i \in \mathcal{I}\left(D_{i}\right)} m_{i j}\left(\mathbf{U}_{j}^{H, n+1}-\mathbf{U}_{j}^{n}\right)-\tau d_{i j}^{H, n}\left(\mathbf{U}_{j}^{n}-\mathbf{U}_{i}^{n}\right)=m_{i}\left(\mathbf{U}_{i}^{L, n+1}-\mathbf{U}_{i}^{n}\right)-\tau d_{i j}^{L, n}\left(\mathbf{U}_{j}^{n}-\mathbf{U}_{i}^{n}\right) .
$$


This equality is rewritten in the following form better suited for post-processing:

$$
m_{i}\left(\mathbf{U}_{j}^{H, n+1}-\mathbf{U}_{j}^{L, n+1}\right)=\sum_{i \in \mathcal{I}\left(D_{i}\right)} \Delta_{i j}\left(\mathbf{U}_{j}^{H, n+1}-\mathbf{U}_{j}^{n}\right)+\tau\left(d_{i j}^{H, n}-d_{i j}^{L, n}\right)\left(\mathbf{U}_{j}^{n}-\mathbf{U}_{i}^{n}\right),
$$

where we have set $\Delta_{i j}:=m_{i} \delta_{i j}-m_{i j}$. The above identity can be rewritten as follows:

$$
\left\{\begin{array}{l}
m_{i}\left(\mathbf{U}^{H, n+1}-\mathbf{U}^{L, n+1}\right)=\sum_{j \in \mathcal{I}\left(D_{i}\right)} \boldsymbol{A}_{i j}^{n} \\
\boldsymbol{A}_{i j}^{n}:=\Delta_{i j}\left(\mathbf{U}_{j}^{H, n+1}-\mathbf{U}_{j}^{n}-\left(\mathbf{U}_{i}^{H, n+1}-\mathbf{U}_{i}^{n}\right)\right)-\tau\left(d_{i j}^{H, n}-d_{i j}^{L, n}\right)\left(\mathbf{U}_{j}^{n}-\mathbf{U}_{i}^{n}\right),
\end{array}\right.
$$

where we used $\sum_{j \in \mathcal{I}\left(D_{i}\right)} \Delta_{i j}=0$. Observe that the matrix $\boldsymbol{A}^{n}$ is skew-symmetric; the immediate consequence is that $\sum_{i \in \mathcal{I}} m_{i} \mathbf{U}_{j}^{H, n+1}=\sum_{i \in \mathcal{I}} m_{i} \mathbf{U}_{j}^{L, n+1}$, i.e., the total mass of the provisional high-order solution is the same at that of the low-order solution.

The next step consists of introducing symmetric limiting parameters $\ell_{i j}=\ell_{j i} \in$ $[0,1]$ and estimating $\ell_{i j}$ so that the new quantity $\mathbf{U}^{n+1}=\mathbf{U}^{L, n+1}+\frac{1}{m_{i}} \sum_{j \in \mathcal{I}\left(D_{i}\right)} \ell_{i j} \boldsymbol{A}_{i j}^{n}$ satisfies the expected bounds. Note again that the skew-symmetry of $\boldsymbol{A}^{n}$ together with the symmetry of the limiter implies that $\sum_{i \in \mathcal{I}} m_{i} \mathbf{U}_{j}^{n+1}=\sum_{i \in \mathcal{I}} m_{i} \mathbf{U}_{j}^{L, n+1}$ for any choice of limiter $\ell_{i j}$, i.e., the limiting process is conservative. Using the notation introduced at the end of $\S 4.1$, we seek $\ell_{i j}$ so that $\Psi\left(\mathbf{U}^{n+1}\right) \geq 0$.

We now depart form the FCT algorithm as described in [45] by introducing $\lambda_{j}:=$ $\frac{1}{\operatorname{card}\left(\mathcal{I}\left(D_{i}\right)\right)-1}, j \in \mathcal{I}\left(D_{i}\right) \backslash\{i\}$, and rewriting (4.5) as follows

$$
\mathbf{U}_{i}^{n+1}=\sum_{j \in \mathcal{I}\left(D_{i}\right) \backslash\{i\}} \lambda_{j}\left(\mathbf{U}_{i}^{L, n+1}+\ell_{i j} \mathbf{P}_{i j}\right), \quad \text { with } \quad \mathbf{P}_{i j}:=\frac{1}{m_{i} \lambda_{j}} \mathbf{A}_{i j}^{n} .
$$

Note that $\mathbf{U}_{i}^{n+1}=\mathbf{U}_{i}^{L, n+1}$ if $\ell_{i j}=0$ and $\mathbf{U}_{i}^{n+1}=\mathbf{U}_{i}^{H, n+1}$ if $\ell_{i j}=1$. The following lemma is the driving force of the limiting technique that we propose.

Lemma 4.3. Let $\Psi(\boldsymbol{u}): \mathcal{A} \rightarrow \mathbb{R}$ be a quasiconcave function. Assume that the limiting parameters $\ell_{i j} \in[0,1]$ are such that be such that $\Psi\left(\mathbf{U}_{i}^{L, n+1}+\ell_{i j} \mathbf{P}_{i j}\right) \geq 0$, $i \neq j \in \mathcal{I}\left(D_{i}\right)$, then the following inequality holds true:

$$
\Psi\left(\sum_{j \in \mathcal{I}\left(D_{i}\right) \backslash\{i\}} \lambda_{j}\left(\mathbf{U}_{i}^{L, n+1}+\ell_{i j} \mathbf{P}_{i j}\right)\right) \geq 0 .
$$

Proof. Let $L_{0}=\{\mathbf{U} \in \mathcal{A} \mid \psi(\mathbf{U}) \geq 0\}$. By definition all the limited states $\mathbf{U}_{i}^{L, n+1}+$ $\ell_{i j} \mathbf{P}_{i j}$ are in $L_{0}$ for all $i \neq j \in \mathcal{I}\left(D_{i}\right)$. Since $\Psi$ is quasiconcave, the upper level set $L_{0}$ is convex. As a result, the convex combination $\sum_{j \in \mathcal{I}\left(D_{i}\right) \backslash\{i\}} \lambda_{j}\left(\mathbf{U}_{i}^{L, n+1}+\ell_{i j} \mathbf{P}_{i j}\right)$ is in $L_{0}$, i.e., $\Psi\left(\sum_{j \in \mathcal{I}\left(D_{i}\right) \backslash\{i\}} \lambda_{j}\left(\mathbf{U}_{i}^{L, n+1}+\ell_{i j} \mathbf{P}_{i j}\right)\right) \geq 0$, which concludes the proof.

Lemma 4.4. Let $\ell_{j}^{i}$ be defined by

$$
\ell_{j}^{i}= \begin{cases}1 & \text { if } \Psi\left(\mathbf{U}_{i}^{L, n+1}+\mathbf{P}_{i j}\right) \geq 0 \\ \max \left\{\ell \in[0,1] \mid \Psi\left(\mathbf{U}_{i}^{L, n+1}+\ell \mathbf{P}_{i j}\right) \geq 0\right\} & \text { otherwise, }\end{cases}
$$

for every $i \in \mathcal{I}$ and $j \in \mathcal{I}\left(D_{i}\right)$. The following two statements hold true: (i) $\Psi\left(\mathbf{U}_{i}^{L, n+1}+\right.$ $\left.\ell \mathbf{P}_{i j}\right) \geq 0$ for every $\ell \in\left[0, \ell_{j}^{i}\right]$; (ii) In particular, setting $\ell_{i j}=\min \left(\ell_{j}^{i}, \ell_{i}^{j}\right)$, we have $\Psi\left(\mathbf{U}_{i}^{L, n+1}+\ell_{i j} \mathbf{P}_{i j}\right) \geq 0$ and $\ell_{i j}=\ell_{j i}$ for every $i \in \mathcal{I}$ and $j \in \mathcal{I}\left(D_{i}\right)$. 
Proof. (i) First, if $\Psi\left(\mathbf{U}_{i}^{L, n+1}+\mathbf{P}_{i j}\right) \geq 0$ we observe that $\Psi\left(\mathbf{U}_{i}^{L, n+1}+\ell \mathbf{P}_{i j}\right) \geq 0$ for any $\ell \in[0,1]$ because $\mathbf{U}_{i}^{L, n+1} \in L_{0}(\Psi), \mathbf{U}_{i}^{L, n+1}+\mathbf{P}_{i j} \in L_{0}(\Psi)$ and $L_{0}(\Psi)$ is convex. Second, if $\Psi\left(\mathbf{U}_{i}^{L, n+1}+\mathbf{P}_{i j}\right)<0$, we observe that $\ell_{j}^{i}$ is uniquely defined and for any $\ell \in\left[0, \ell_{j}^{i}\right]$ we have $\Psi\left(\mathbf{U}_{i}^{L, n+1}+\ell \mathbf{P}_{i j}\right) \geq 0$ because $\mathbf{U}_{i}^{L, n+1} \in L_{0}(\Psi), \mathbf{U}_{i}^{L, n+1}+\ell_{j}^{i} \mathbf{P}_{i j} \in$ $L_{0}(\Psi)$ and $L_{0}(\Psi)$ is convex. (ii) Since $\ell_{i j}=\min \left(\ell_{j}^{i}, \ell_{i}^{j}\right) \leq \ell_{j}^{i}$, the above construction implies that $\Psi\left(\mathbf{U}_{i}^{L, n+1}+\ell_{i j} \mathbf{P}_{i j}\right) \geq 0$. Note finally that $\ell_{i j}=\min \left(\ell_{j}^{i}, \ell_{i}^{j}\right)=\ell_{j i}$.

Remark 4.5 (Extension to general hyperbolic systems). Notice that Lemma 4.3 and Lemma 4.4 are not specific to the Euler equations. These results can be used to limit solutions of arbitrary hyperbolic systems where the invariant domain is described by quasiconcave constraints.

4.3. Application to the Euler equations. We explain in this section how to use Lemma 4.3 and Lemma 4.4 to enforce the quasiconcave constraints described in $\S 4.1$. The algorithm goes as follows:

(i) Given the state $\mathbf{U}^{n}$, which we assume to be admissible, we compute $\mathbf{U}^{L, n+1}$ and $\mathbf{U}^{H, n+1}$ as explained in $\S 3.1$ and either $\S 3.3$ or 3.4 ;

(ii) The density is limited by invoking Lemma 4.3 and Lemma 4.4 and the bounds described in $\S 4.1$ to enforce the quasiconcave constraints $\Psi(\mathbf{U})=\rho-\rho_{i}^{\min } \geq 0$ and $\Psi(\mathbf{U})=\rho_{i}^{\max }-\rho \geq 0$. The resulting limiter is denoted by $\ell_{i j}^{\rho}$ and details of the computation of $\ell_{i j}^{\rho}$ are given in $\S 4.4$;

(iii) The internal energy $\rho e:=E-\frac{\boldsymbol{m}^{2}}{2 \rho}$ is limited by invoking Lemma 4.3 and Lemma 4.4 to enforce the quasiconcave constraint $\Psi(\mathbf{U})=E-\frac{m^{2}}{2 \rho}-\varepsilon_{i}^{\min } \geq 0$. The corresponding limiter is denoted $\ell_{i j}^{e} \leq \ell_{i j}^{\rho}$ and details of the computation of $\ell_{i j}^{e}$ are given in $\S 4.5$;

(iv) The minimum principle on the specific entropy is enforced by using $\Psi(\mathbf{U})=$ $\Phi(\mathbf{U})-s_{i}^{\min }$. The details on the computation of the corresponding limiter $\ell_{i j}^{s} \leq \ell_{i j}^{e}$ are given in $\S 4.6$.

(v) Finally, upon setting $\ell_{i j}:=\ell_{i j}^{s}$, the update $\mathbf{U}^{n+1}$ is computed by setting $\mathbf{U}^{n+1}=\mathbf{U}^{L, n+1}+\frac{1}{m_{i}} \sum_{j \in \mathcal{I}\left(D_{i}\right)} \ell_{i j} \boldsymbol{A}_{i j}^{n}$. This type of limitation can be iterated a few times by observing that

$$
\mathbf{U}^{H, n+1}=\mathbf{U}^{L, n+1}+\frac{1}{m_{i}} \sum_{j \in \mathcal{I}\left(D_{i}\right)} \ell_{i j} \boldsymbol{A}_{i j}^{n}+\frac{1}{m_{i}} \sum_{j \in \mathcal{I}\left(D_{i}\right)}\left(1-\ell_{i j}\right) \boldsymbol{A}_{i j}^{n} .
$$

Then setting $\mathbf{U}^{(0)}:=\mathbf{U}^{L, n+1}$ and $\boldsymbol{A}_{i j}^{(0)}:=\boldsymbol{A}_{i j}^{n}$ the iterative algorithm proceeds as shown in Algorithm 1. In the numerical simulations reported at the end of the paper we have taken $k_{\max }=1$.

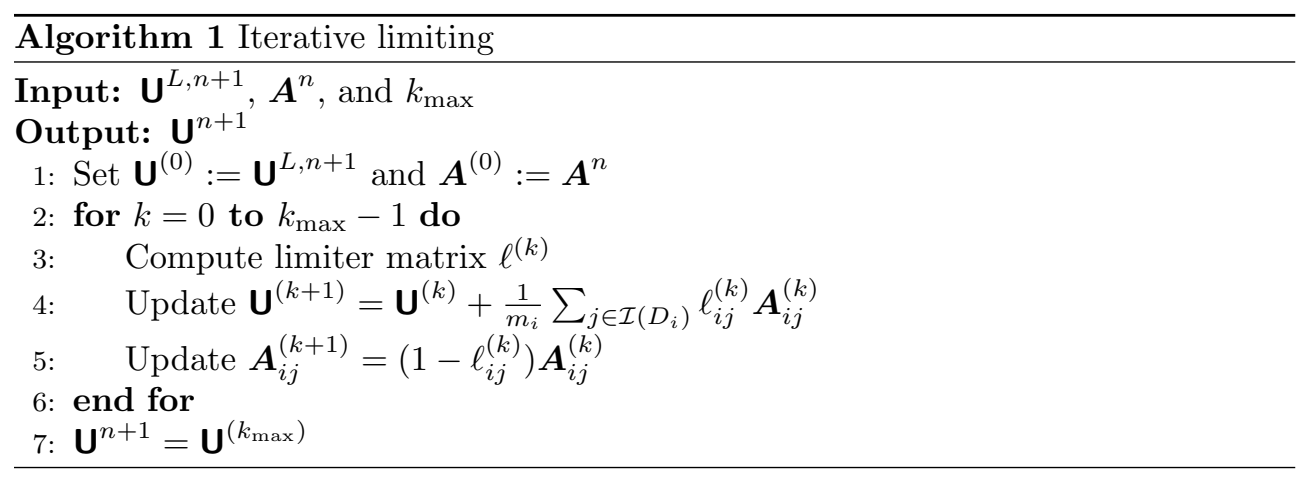


Remark 4.6 (Other quantities). As observed in Section 4.1 it is also possible to impose additional limiting based on quasiconcave constraints. For example, one could limit the total energy from below and from above. Numerical experiments reveal that this extra limitation does not improve the performance of the scheme. All the tests reported in $\S 5$ are done by limiting the density, the internal energy and the specific entropy as described above. We have found that limiting the internal energy delivers second-order accuracy in the maximum norm, but has a tendency to over-dissipate contact discontinuities. Note that limiting the specific entropy amounts in effect to limit the internal energy.

Remark 4.7 (Equation of state). So far, everything we have described is independent of the equation of state.

4.4. Limitation on the density. The limitation on the density as specified by (4.7) proceeds as follows. To avoid divisions by zero, we introduce the small parameter $\epsilon:=10^{-14}$ set $\epsilon_{i}=\epsilon \rho_{i}^{\max }$ for all $i \in \mathcal{I}$. Let us denote by $P_{i j}^{\rho}$ the $\rho$-component of $\boldsymbol{P}_{i j}$, and let us set

$$
\ell_{j}^{i, \rho}= \begin{cases}\min \left(\frac{\left|\rho_{i}^{\min }-\rho_{i}^{L, n+1}\right|}{\left|P_{i j}^{\rho}\right|+\epsilon_{i}}, 1\right) & \text { if } \rho_{i}^{L, n+1}+P_{i j}^{\rho}<\rho_{i}^{\min } \\ 1 & \text { if } \rho_{i}^{\min } \leq \rho_{i}^{L, n+1}+P_{i j}^{\rho} \leq \rho_{i}^{\max } \\ \min \left(\frac{\left|\rho_{i}^{\max }-\rho_{i}^{L, n+1}\right|}{\left|P_{i j}^{\rho}\right|+\epsilon_{i}}, 1\right) & \text { if } \rho_{i}^{\max }<\rho_{i}^{L, n+1}+P_{i j}^{\rho} .\end{cases}
$$

Upon setting $\Psi_{+}(\mathbf{U})=\rho-\rho_{i}^{\min }$, and $\Psi_{-}(\mathbf{U})=\rho_{i}^{\max }-\rho$, we have the following result whose proof is left to the reader.

Lemma 4.8. The definition (4.8) implies that $\Psi_{ \pm}\left(\mathbf{U}_{i}^{L, n+1}+\ell \mathbf{P}_{i j}\right) \geq 0$ for all $\ell \in\left[0, \ell_{j}^{i, \rho}\right]$.

Remark 4.9 (Covolume EOS). In the case of the covolume equation of state, $p(1-b \rho)=(\gamma-1) \rho e$, it is known that $\mathcal{A}=\left\{(\rho, \boldsymbol{m}, E) \mid \rho>0, e>0, s \geq s^{\min }, b \rho<1\right\}$ is an invariant domain, see Guermond and Popov [12, Prop. A.1]. The above method can be used to enforce the additional affine constraint $1-b \rho>0$.

Remark 4.10 (Total energy). The limitation on the total energy can be done exactly as for the density. Let us emphasize though that we have not found this operation to be useful and it is not done in the numerical tests reported at the end of the paper.

4.5. Limitation on the internal energy $\rho e$. In this section we explain how to compute the limiter to enforce the local minimum principle on the internal energy $\rho e$ as stated in (4.4).

Upon setting $\Psi(\mathbf{U}):=(\rho e)(\mathbf{U})-\varepsilon_{i}^{\min }$ with $\mathbf{U}:=(\rho, \boldsymbol{m}, E)$, and by virtue of Lemma 4.3 and Lemma 4.4, we have to estimate $\ell_{j}^{i, e} \in\left[0, \ell_{j}^{i, \rho}\right]$ so that $\Psi\left(\mathbf{U}_{i}^{L, n+1}+\right.$ $\left.\ell \mathbf{P}_{i j}\right) \geq 0$ for all $\ell \in\left[0, \ell_{j}^{i, e}\right]$. We define the auxiliary function $\psi:\{\mathbf{U} \mid \rho>0\} \rightarrow \mathbb{R}$

$$
\psi(\mathbf{U}):=\left(\rho^{2} e\right)(\mathbf{U})-\varepsilon_{i}^{\min } \rho=\rho E-\frac{1}{2} \boldsymbol{m}^{2}-\varepsilon_{i}^{\min } \rho .
$$

Then the above problem is equivalent seeking $\ell_{j}^{i, e} \in\left[0, \ell_{j}^{i, \rho}\right]$ so that $\psi\left(\mathbf{U}_{i}^{L, n+1}+\ell \mathbf{P}_{i j}\right) \geq$ 0 for all $\ell \in\left[0, \ell_{j}^{i, e}\right]$. The key observation is that now $\psi$ is a quadratic functional with

$$
D \psi(\mathbf{U})=\left(\begin{array}{c}
E-\varepsilon_{i}^{\min } \\
-\boldsymbol{m} \\
\rho
\end{array}\right), \quad D^{2} \psi(\mathbf{U})=\left(\begin{array}{ccc}
0 & \mathbf{0}^{\top} & 1 \\
\mathbf{0} & -\mathbb{I} & \mathbf{0} \\
1 & \mathbf{0}^{\top} & 0
\end{array}\right) .
$$


Then upon setting $a:=\frac{1}{2} \mathbf{P}_{i j}^{\top} D^{2} \psi \mathbf{P}_{i j}, b:=D \psi\left(\mathbf{U}_{i}^{L, n+1}\right) \cdot \mathbf{P}_{i j}$ and $c:=\psi\left(\mathbf{U}_{i}^{L, n+1}\right)$, we have

$$
\psi\left(\mathbf{U}_{i}^{L, n+1}+t \mathbf{P}_{i j}\right)=a t^{2}+b t+c .
$$

Let $t_{0}$ be the smallest positive root of the equation $a t^{2}+b t+c=0$, with the convention that $t_{0}=1$ if the equation has no positive root. Then we choose $\ell_{j}^{i, e}$ to be such that

$$
\ell_{j}^{i, e}=\min \left(t_{0}, \ell_{j}^{i, \rho}\right)
$$

Lemma 4.11. The definition (4.9) implies that $\Psi\left(\mathbf{U}_{i}^{L, n+1}+\ell \mathbf{P}_{i j}\right) \geq 0$ for all $\ell \in$ $\left[0, \ell_{j}^{i, e}\right]$.

Proof. If there is no positive root to the equation $a t^{2}+b t+c=0$ and since we have established that $c=\psi\left(\mathbf{U}_{i}^{L, n+1}\right) \geq 0$ (see (4.4)), we have $a t^{2}+b t+c \geq 0$ for all $t \geq 0$; that is, $\Psi\left(\mathbf{U}_{i}^{L, n+1}+\ell \mathbf{P}_{i j}\right) \geq 0$ for all $\ell \geq 0$, and in particular this is true for all $\ell \in$ $\left[0, \ell_{j}^{i, e}\right]$. Otherwise, if there is at least one positive root to the equation $a t^{2}+b t+c=0$, then denoting by $t_{0}$ the smallest positive root, we have $a t^{2}+b t+c \geq 0$ for all $t \in\left[0, t_{0}\right]$ (if not, there would exist $t_{1} \in\left(0, t_{0}\right)$ s.t. $a t_{1}^{2}+b t_{1}+c<0$ and the intermediate value theorem would imply the existence a root $t^{*} \in\left(0, t_{1}\right)$ which contradict that $t_{0}$ is the smallest positive root). This implies that $\Psi\left(\mathbf{U}_{i}^{L, n+1}+\ell \mathbf{P}_{i j}\right) \geq 0$ for all $\ell \in\left[0, t_{0}\right]$, and in particular this is true for all $\ell \in\left[0, \ell_{j}^{i, e}\right]$ owing to (4.9).

Remark 4.12 (Equation of state). Observe that the proposed limitation on $\rho$ is independent of the equation of state.

4.6. Minimum principle on the specific entropy. We now describe how to compute the limiter to enforce the local minimum principle on the specific entropy. Khobalatte and Perthame [25] is the first paper we are aware of where this type of limiting is done.

By virtue of Lemma 4.3 and Lemma 4.4, we have to estimate $\ell_{j}^{i, s} \in\left[0, \ell_{j}^{i, e}\right]$ so that $\Psi\left(\mathbf{U}_{i}^{L, n+1}+\ell \mathbf{P}_{i j}\right) \geq 0$ for all $\ell \in\left[0, \ell_{j}^{i, s}\right]$, with $\Psi(\mathbf{U}):=\Phi(\mathbf{U})-s_{i}^{\min }$, where we recall that $\Phi(\mathbf{U}):=s(\rho, e(\mathbf{U}))$ is the specific entropy as a function of the conservative variables.

Lemma 4.13. Let $t_{0}$ be defined as follows: (i) If $\Psi\left(\mathbf{U}_{i}^{L, n+1}+\mathbf{P}_{i j}\right) \geq 0$, then we set $t_{0}=1$; (ii) If $\Psi\left(\mathbf{U}_{i}^{L, n+1}+\mathbf{P}_{i j}\right)<0$ and $\Psi\left(\mathbf{U}_{i}^{L, n+1}\right)>0$, we set $t_{0}$ to be the unique positive root to the equation $\Psi\left(\mathbf{U}_{i}^{L, n+1}+t \mathbf{P}_{i j}\right)=0$; (iii) If $\Psi\left(\mathbf{U}_{i}^{L, n+1}+\mathbf{P}_{i j}\right)<$ 0 and $\Psi\left(\mathbf{U}_{i}^{L, n+1}\right)=0$, the equation $\Psi\left(\mathbf{U}_{i}^{L, n+1}+t \mathbf{P}_{i j}\right)=0$ has exactly two roots (possibly equal) and we take $t_{0}$ to be the largest nonnegative root. More precisely, if $D \psi\left(\mathbf{U}_{i}^{L, n+1}\right) \cdot \mathbf{P}_{i j} \leq 0$ then $t_{0}=0$, and if $D \psi\left(\mathbf{U}_{i}^{L, n+1}\right) \cdot \mathbf{P}_{i j}>0$ then $t_{0}>0$ is the unique positive root of $\Psi\left(\mathbf{U}_{i}^{L, n+1}+t \mathbf{P}_{i j}\right)=0$ and has to be computed. Then setting $\ell_{j}^{i, s}=\min \left(t_{0}, \ell_{j}^{i, e}\right)$, we have $\Psi\left(\mathbf{U}_{i}^{L, n+1}+\ell \mathbf{P}_{i j}\right) \geq 0$ for all $\ell \in\left[0, \ell_{j}^{i, s}\right]$.

Proof. Let us first observe that the equation $\Psi\left(\mathbf{U}_{i}^{L, n+1}+t \mathbf{P}_{i j}\right)=0$ has exactly two roots (possibly equal) because the upper level set $L_{0}=\{\mathbf{U} \mid \Psi(\mathbf{U}) \geq 0$ is convex and any line that intersects the upper level set crosses the boundary at two points, say $t_{-} \leq t_{+},\left(t_{-}=t_{+}\right.$when the line is tangential to the boundary of the upper level set). Note that $t_{-} \leq 0$ since $\Psi\left(\mathbf{U}_{i}^{L, n+1}\right) \geq 0$. (i) If $\Psi\left(\mathbf{U}_{i}^{L, n+1}+\mathbf{P}_{i j}\right) \geq 0$, then $t_{+} \geq 1$ and the entire segment $\left\{\mathbf{U}_{i}^{L, n+1}+t \mathbf{P}_{i j} \mid t \in\left[0, t_{0}=1\right]\right\}$ in $L_{0}$ by convexity. (ii) If $\Psi\left(\mathbf{U}_{i}^{L, n+1}+\mathbf{P}_{i j}\right)<0$ and $\Psi\left(\mathbf{U}_{i}^{L, n+1}\right)>0$, then $t_{+} \in(0,1)$, and upon setting $t_{0}=t_{+}$, the entire segment $\left\{\mathbf{U}_{i}^{L, n+1}+t \mathbf{P}_{i j} \mid t \in\left[0, t_{0}\right]\right\}$ in $L_{0}$ by convexity. (iii) If 
$\Psi\left(\mathbf{U}_{i}^{L, n+1}+\mathbf{P}_{i j}\right)<0$ and $\Psi\left(\mathbf{U}_{i}^{L, n+1}\right)=0$, there are two possibilities: (i) $D \psi\left(\mathbf{U}_{i}^{L, n+1}\right)$. $\mathbf{P}_{i j} \leq 0$; and (ii) $D \psi\left(\mathbf{U}_{i}^{L, n+1}\right) \cdot \mathbf{P}_{i j}>0$. If $D \psi\left(\mathbf{U}_{i}^{L, n+1}\right) \cdot \mathbf{P}_{i j} \leq 0$ then by convexity $\Psi\left(\mathbf{U}_{i}^{L, n+1}+t \mathbf{P}_{i j}\right)<0$ for all $t>0$. Hence, $t_{+}=0$ is the largest nonnegative toot of the equation $\Psi\left(\mathbf{U}_{i}^{L, n+1}+t \mathbf{P}_{i j}\right)=0$ and therefore $t_{0}=t_{+}=0$. In the remaining case, $D \psi\left(\mathbf{U}_{i}^{L, n+1}\right) \cdot \mathbf{P}_{i j}>0$, we have that $0<t_{+}<1$ and $t_{0}=t_{+}$.

Let us now explain how the above line search can be done efficiently. Thermodynamic principles imply that there exists a smooth function $g: \mathbb{R}_{+} \times \mathbb{R} \rightarrow \mathbb{R}_{+}$such that $\rho e=g(s, \rho)$. Note that the identity $\partial_{s} e(\rho, s)=\frac{1}{\partial_{e} s(\rho, e)}$ together with the fundamental thermodynamic inequality $\partial_{e} s(\rho, e)>0$, which is equivalent to the temperature being positive, implies $\partial_{s} g(\rho, s)>0$. Since $\partial_{s} g>0$, the minimum principle on the specific entropy $\Psi(\mathbf{U}):=\Phi(\mathbf{U})-s_{i}^{\min } \geq 0$ is equivalent to enforcing $g(s, \rho)=\rho e \geq g\left(s_{i}^{\min }, \rho\right)$.

When the function $g(s, \rho)$ satisfies $\partial_{\rho \rho} g \geq 0$ and up to a change of notation, the above constraint can be reformulated as follows: $\Psi(\mathbf{U}):=\rho e(\mathbf{U})-g\left(s_{i}^{\min }, \rho\right) \geq 0$. Note that $\partial_{\rho \rho} g \geq 0$ implies that the new function $\Psi(\mathbf{U})$ is concave with respect to the conservative variables; as a result, the line search $h(t):=\psi\left(\mathbf{U}_{i}^{L, n+1}+t \mathbf{P}_{i j}\right)=0$ can be done efficiently because $h$ is concave and $h(0) \geq 0$. If $h(1) \geq 0$ we set $t_{0}=1$ and if $h(1)<0$ we use a combination of secant and Newton method to find the unique $0 \leq t_{0}<1$ such that $h\left(t_{0}\right)=0$. For example, the covolume equation of state falls into this category since in this case we have $\rho e=\frac{\rho^{\gamma}}{(1-b \rho)^{\gamma-1}} \exp \left((\gamma-1)\left(s-s_{0}\right)\right)=: g(s, \rho)$. This is also the case for the stiffened gas equation of state, $\rho e=e_{0} \rho+p_{\infty}(1-$ $b \rho)+\frac{\rho^{\gamma}}{(1-b \rho)^{\gamma-1}} \exp \left((\gamma-1)\left(s-s_{0}\right)\right)=: g(s, \rho)$ where $e_{0}, s_{0}$ and $p_{\infty}$ are are constant coefficients characteristic of the thermodynamic properties of the fluid, see Metayer and Saurel [33] for details.

In the general case, i.e., when $g(s, \rho)$ does not satisfy $\partial_{\rho \rho} g \geq 0$, we can use a different strategy for imposing $\Psi(\mathbf{U})=\Phi(\mathbf{U})-s_{i}^{\min } \geq 0$. Namely, using that $\rho>0$ and using again a change of notation, we transform the constraint to $\Psi(\mathbf{U}):=$ $\rho \Phi(\mathbf{U})-s_{i}^{\min } \rho \geq 0$. Note that the function $-\rho \Phi(\mathbf{U})$ is a mathematical entropy for the Euler system and under the standard assumptions (hyperbolicity and positive temperature) it is convex, see Harten et al. [19, Thm. 2.1]. Therefore, the line search $h(t):=\psi\left(\mathbf{U}_{i}^{L, n+1}+t \mathbf{P}_{i j}\right)=0$ can be done efficiently because $h$ is concave and $h(0) \geq 0$. If $h(1) \geq 0$ we set $t_{0}=1$ and if $h(1)<0$ we use a combination of secant and Newton method to find the unique $0 \leq t_{0}<1$ such that $h\left(t_{0}\right)=0$.

4.7. Relaxation. It is observed in Khobalatte and Perthame [25, §3.3] that strictly enforcing the minimum principle on the specific entropy degrades the converge rate to first-order; it is said therein that "It seems impossible to perform secondorder reconstruction satisfying the conservativity requirements ... and the maximum principle on $S$ ". We have also observed this phenomenon. Moreover, it is well known that, when applied to scalar conservation equations, limitation (in some broad sense) reduces the accuracy to first-order near maxima and minima of the solution. One typical way to address this issue in the finite volume literature consists of relaxing the slope reconstructions; see Harten and Osher [18], Schmidtmann et al. [37, §2.1]. In the present context, since we do not have any slope to reconstruct, we are going to relax the constraints so that the violation of the constraint is second-order accurate.

4.7.1. Relaxation on the density and the internal energy. Let us denote by $\varrho$ one of the quantities that we may want to limit from below, excluding the specific entropy, say $\rho,-\rho$, or $(\rho e)$ and let $\varrho^{\text {min }}$ be the corresponding bound given by the technique described in $\S 4$.1. For each $i \in \mathcal{I}$, we set $\Delta^{2} \varrho_{i}^{n}:=\sum_{i \neq j \in \mathcal{I}\left(D_{i}\right)} \varrho_{i}^{n}-\varrho_{j}^{n}$ 
and we define

$$
\begin{aligned}
& \overline{\Delta^{2} \varrho_{i}^{n}}:=\frac{1}{2 \operatorname{card}\left(\mathcal{I}\left(D_{i}\right)\right)} \sum_{i \neq j \in \mathcal{I}\left(D_{i}\right)}\left(\frac{1}{2} \Delta^{2} \varrho_{i}^{n}+\frac{1}{2} \Delta^{2} \varrho_{j}^{n}\right), \\
& \widetilde{\Delta^{2} \varrho_{i}^{n}}:=\operatorname{minmod}\left\{\frac{1}{2} \Delta^{2} \varrho_{j}^{n} \mid j \in \mathcal{I}\left(D_{i}\right)\right\},
\end{aligned}
$$

where the minmod function of a finite set is defined to be zero if there are two numbers of different sign in this set, and it is equal to the number whose absolute value is the smallest otherwise. Then we propose two types of relaxation defined as follows:

$$
\begin{gathered}
\overline{\varrho_{i}^{\min }}=\max \left(0.99 \varrho_{i}^{\min }, \varrho_{i}^{\min }-\left|\overline{\Delta^{2} \varrho_{i}^{n}}\right|\right) \\
\varrho_{i}^{\min }=\max \left(0.99 \varrho_{i}^{\min }, \varrho_{i}^{\min }-\left|\widetilde{\Delta^{2} \varrho_{i}^{n}}\right|\right) .
\end{gathered}
$$

When doing limitation we use either $\overline{\varrho_{i}^{\min }}$ or $\widetilde{\varrho_{i}^{\min }}$ instead of $\varrho_{i}^{\text {min }}$. It is shown in the numerical section that both relaxations are robust.

Remark 4.14 (relaxation vs. no relaxation). We have observed numerically that the proposed method is second-order accurate in the $L^{1}$-norm without relaxation if limitation is done on the density and the internal energy. Relaxation is necessary only to get second-order accuracy in the $L^{\infty}$-norm. We have observed though that the minmod relaxation is slightly more restrictive than the other one since it does not deliver second-order accuracy in the maximum norm; only the averaging relaxation (4.12) has been found to give second-order in the $L^{\infty}$-norm.

Remark 4.15 (positivity). Note that the somewhat ad hoc threshold $0.99 \varrho_{i}^{\min }$ in the above definitions imply that one never relaxes more than $1 \%$ of the legitimate lower bound. In particular, when applied to the density or the internal energy the above relaxation guarantees positivity of the density and the internal energy.

4.7.2. Relaxation on the specific entropy. We proceed as in Khobalatte and Perthame $[25, \S 3.3]$ and relax the lower bound on the specific entropy more aggressively since in smooth regions this function is constant. Let $\varrho$ be the quantity associated with the constraint on the specific entropy; it could be $s, \exp (s)$, or $\rho e / \rho^{\gamma}$ in the case of polytropic gases, depending on the way one chooses to enforce the minimum principle on the specific entropy (see $\S 4.6)$. Let $\boldsymbol{x}_{i j}=\frac{1}{2}\left(\boldsymbol{x}_{i}+\boldsymbol{x}_{j}\right)$. We measure the local variations of $\varrho$ by setting $\Delta \varrho_{i}^{n}=\max _{i \neq j \in \mathcal{I}\left(D_{i}\right)}\left(\varrho^{n}\left(\boldsymbol{x}_{i j}\right)-\varrho_{i}^{\text {min }}\right)$ and we relax $\varrho_{i}^{\text {min }}$ by setting

$$
\overline{\varrho_{i}^{\min }}=\max \left(0.99 \varrho_{i}^{\min }, \varrho_{i}^{\min }-\Delta \varrho_{i}^{n}\right) .
$$

Note that contrary to the appearances, and as already observed in [25], the size of the relaxation is $\mathcal{O}\left(h^{2}\right)$ : In the vicinity of shocks, there is no need for relaxation since the first-order viscosity takes over and thereby makes the solution minimum principle preserving on the specific entropy. In smooth regions, i.e., isentropic regions, the specific entropy is constant and $\Delta \varrho_{i}^{n}$ measures the local curvature of $s$ induced by the nonlinearity of $s$, that is to say $\Delta \varrho_{i}^{n}$ is $\mathcal{O}\left(h^{2}\right)$.

Remark 4.16 (positivity). The threshold $0.99 \varrho_{i}^{\min }$ guarantees positivity of the internal energy. We have observed numerically that this threshold is never reached when the mesh is reasonably fine enough. 
5. Numerical illustrations. We report in this section numerical tests we have done to illustrate the performance of the proposed method. All the tests are done with the equation of state $p=(\gamma-1) \rho e$, i.e., $(\gamma-1) s(\rho, e)=\exp \left(\rho e / \rho^{\gamma}\right)$.

5.1. Technical details. Three different codes implementing the method described in the paper have been written to ensure reproducibility. Limiting is done only once in the three codes, i.e., $k_{\max }=1$.

The first one, henceforth called Code 1, does not use any particular software. It is based on Lagrange elements on simplices. This code has been written to be dimension-independent, i.e., the same data structure and subroutines are used in one dimension and in two dimensions. The two-dimensional meshes used for Code 1 are nonuniform triangular Delaunay meshes. All the computations reported in the paper are done with continuous $\mathbb{P}_{1}$ elements. The high-order method uses the entropy viscosity commutator described in (3.13)-(3.14) with the entropy $p^{\frac{1}{\gamma}}$. Limitation on the density is enforced by using the technique described in $\S 4.4$. The bounds on the density are relaxed by using the averaging technique described in $\S 4.7$. The minimum principle on the specific entropy $\exp ((\gamma-1) s) \geq \exp \left((\gamma-1) s^{\min }\right)$ is enforced by using the method described in $\S 4.6$ with the constraint $\Psi(\mathbf{U}):=\rho e-\varrho^{\min } \rho^{\gamma} \geq 0$, where we recall that $\rho e / \rho^{\gamma}=\exp ((\gamma-1) s)$. The lower bound on the specific entropy is defined by using $\varrho_{i}^{\min }=\min _{j \in \mathcal{I}\left(D_{i}\right)} \rho_{i}^{n} e_{i}^{n} /\left(\rho_{i}^{n}\right)^{\gamma}$ (instead of (4.3)) and $\varrho_{i}^{\min }$ is relaxed by using (4.14) with $\varrho=\rho e / \rho^{\gamma}$. No limitation on the internal energy is applied in Code 1; the positivity of the internal energy is guaranteed by the minimum principle on the specific entropy.

The second code, henceforth called Code 2, is based on the open-source finite element library deal.II, see Arndt et al. [1], Bangerth et al. [2]. The tests reported in the paper are done with continuous $\mathbb{Q}_{1}$ (quadrilateral) elements. The code is written in a dimension-independent fashion and all the computational tasks (e.g., assembly, linear solvers and output) are implemented in parallel via MPI. This code implements the smoothness-based high-order graph viscosity described in $\S 3.3$. All the computations reported in the paper are done with $g(\boldsymbol{u})=S(\boldsymbol{u})$ and $\psi(\alpha)=$ $\alpha^{4}$. (We have verified that the other choices for $g(\boldsymbol{u})$ mentioned in $\S 3.3$ produce comparable results.) As stated at the beginning of $\S 3.3$, this method introduces additional diffusion close to local extrema (whether smooth or not). While this does not affect the second-order decay rates in the $L^{1}$-norm, it degrades the accuracy in the $L^{\infty}$-norm. Limitation is done on the density and the specific entropy exactly as explained above for Code 1 . The minimum principle on the specific entropy is relaxed as explained in $\S 4.7 .2$, but no relaxation is applied on the density bounds.

The last code, henceforth called Code 3, uses the open-source finite element library FEniCS, see e.g., Logg et al. [30], and the computations are done on simplices. The implementation in FEniCS is independent of the space dimension and the polynomial degree of the approximation. The library is fully parallel. All the numerical integrations are done exactly by automatically determining the quadrature degree with respect to the complexity of the underlying integrand and the polynomial space. The results reported in the paper use the entropy viscosity method with the entropy $p^{\frac{1}{\gamma}}$. The limitation and bound relaxation is done on the density and the specific entropy exactly like in Code 1 . We refer the reader to the description of Code 1 for the details.

The time stepping is done in the three codes by using the $\operatorname{SSP}(3,3)$ method (three stages, third-order), see Shu and Osher [39, Eq. (2.18)] and Kraaijevanger [26, Thm. 9.4]. The time step is recomputed at each time step by using the formula 
$\tau=\mathrm{CFL} \times \min _{i \in \mathcal{I}} \frac{m_{i}}{\left|d_{i i}^{L, n}\right|}$ with $d_{i i}^{L, n}=-\sum_{i j} d_{i j}^{L, n}$ given in (3.2).

When working with manufactured solutions, for $p \in[1, \infty]$, we compute a consolidated error indicator at time $t$ by adding the relative error in the $L^{p}$-norm of the density, the momentum, and the total energy as follows:

$$
\delta_{p}(t):=\frac{\left\|\rho_{h}(t)-\rho(t)\right\|_{L^{p}(D)}}{\|\rho(t)\|_{L^{p}(D)}}+\frac{\left\|\boldsymbol{m}_{h}(t)-\boldsymbol{m}(t)\right\|_{\boldsymbol{L}^{p}(D)}}{\|\boldsymbol{m}(t)\|_{\boldsymbol{L}^{p}(D)}}+\frac{\left\|E_{h}(t)-E(t)\right\|_{L^{p}(D)}}{\|E(t)\|_{L^{p}(D)}} .
$$

As some tests may exhibit superconvergence effects we also consider the fully discrete consolidated error indicator $\delta_{p}^{\Pi}(t)$ defined as above with $\rho(t), \boldsymbol{m}(t)$, and $E(t)$ replaced by $\Pi_{h} \rho(t), \Pi_{h} \boldsymbol{m}(t)$, and $\Pi_{h} E(t)$, where $\Pi_{h}$ is the Lagrange interpolation operator.

5.2. 1D smooth wave. We start with a one-dimensional test whose purpose is to estimate the convergence rate of the method with a very smooth solution. We consider the following exact solution to the Euler equations: $v(x, t)=1, p(x, t)=1$ and

$$
\rho(x, t)= \begin{cases}1+2^{6}\left(x_{1}-x_{0}\right)^{-6}\left(x-t-x_{0}\right)^{3}\left(x_{1}-x+t\right)^{3} & \text { if } x_{0} \leq x-t<x_{1} \\ 1 & \text { otherwise }\end{cases}
$$

with $x_{0}=0.1, x_{1}=0.3$ and $\gamma=\frac{7}{5}$. The computational domain is $D=(0,1)$ and the computation is done from $t=0$ to $t=0.6$. The consolidated error indicator in the maximum norm $\delta_{\infty}(t)$ is reported in Table 5. Note that we report the discrete error indicator $\delta_{\infty}^{\Pi}(t)$ for Code 1 (based on the Entropy Viscosity Method) in order to show that we obtain $\mathcal{O}\left(h^{3}\right)$ super-convergence in compliance with the theoretical result stated in Guermond and Pasquetti [10, Prop 2.2]. Code 2, which we recall is based in the smoothness of the mathematical entropy, delivers $\mathcal{O}\left(h^{1.5}\right)$ as expected due to clipping effects induced by the smoothness indicator.

TABLE 1

$1 D$ smooth wave, $\mathbb{P}_{1}$ meshes, Convergence tests with Code 1 and Code 2, CFL $=0.25$.

\begin{tabular}{|c|c|c|c|c|}
\hline \multirow{2}{*}{$\#$ dofs } & \multicolumn{2}{|c|}{ Code 1} & \multicolumn{2}{c|}{ Code 2} \\
\cline { 2 - 5 } & $\delta_{\infty}^{\Pi}(t)$ & rate & $\delta_{\infty}(t)$ & rate \\
\hline 100 & $6.34 \mathrm{E}-02$ & & $2.32 \mathrm{E}-01$ & \\
\hline 200 & $1.62 \mathrm{E}-02$ & 1.96 & $8.30 \mathrm{E}-02$ & 1.48 \\
\hline 400 & $2.69 \mathrm{E}-03$ & 2.59 & $2.87 \mathrm{E}-02$ & 1.53 \\
\hline 800 & $3.74 \mathrm{E}-04$ & 2.85 & $9.66 \mathrm{E}-03$ & 1.57 \\
\hline 1600 & $4.62 \mathrm{E}-05$ & 3.02 & $3.22 \mathrm{E}-03$ & 1.58 \\
\hline 3200 & $5.89 \mathrm{E}-06$ & 2.97 & $1.07 \mathrm{E}-03$ & 1.58 \\
\hline 6400 & $7.37 \mathrm{E}-07$ & 3.00 & $3.74 \mathrm{E}-04$ & 1.52 \\
\hline
\end{tabular}

5.3. Rarefaction wave. We now consider a Riemann problem with a solution whose components are all continuous and whose derivatives have bounded variations. The best-approximation error in the $L^{1}$-norm on quasi-uniform meshes is then $\mathcal{O}\left(h^{2}\right)$. The Riemann problem in question has the following data: $\left(\rho_{L}, v_{L}, p_{L}\right)=$ $\left(3, c_{L}, 1\right),\left(\rho_{R}, v_{R}, p_{R}\right)=\left(\frac{1}{2}, v_{L}+\frac{2}{\gamma-1}\left(c_{L}-c_{R}\right), p_{L}\left(\frac{\rho_{R}}{\rho_{L}}\right)^{\gamma}\right)$, where $c_{L}=\sqrt{\gamma p_{L} / \rho_{L}}$, $c_{R}=\sqrt{\gamma p_{R} / \rho_{R}}$. The equation of state is a gamma-law with $\gamma=\frac{7}{5}$. The exact solution to this problem is a rarefaction wave which can be constructed analytically, see e.g., Toro $[42, \S 4.4]$. The solution is given in Table 2 . In this table, the ratio $\xi:=\frac{x-x_{0}}{t}$ is the self-similar variable, where $x_{0}$ is the location of the discontinuity at $t=0$. This 
problem is quite challenging for any method enforcing the minimum principle on the specific entropy. We have observed that the convergence rate on this problem reduces to $\mathcal{O}(h)$ if the minimum on the specific entropy is not relaxed (see also Khobalatte and Perthame $[25, \S 3.3])$.

TABLE 2

Solution to the rarefaction wave.

\begin{tabular}{|c|c|c|c|}
\hline & $\xi \leq v_{L}-c_{L}$ & $v_{L}-c_{L}<\xi \leq v_{R}-c_{R}$ & $v_{R}-c_{R}<\xi$ \\
\hline$\rho$ & $\rho_{L}$ & $\rho_{L}\left(\frac{2}{\gamma+1}+\frac{\gamma-1}{\gamma+1} \frac{v_{L}-\xi}{c_{L}}\right)^{\frac{2}{\gamma-1}}$ & $\rho_{R}$ \\
\hline$v$ & $v_{L}$ & $\frac{2}{\gamma+1}\left(c_{L}+\frac{\gamma-1}{2} v_{L}+\xi\right)$ & $v_{R}$ \\
\hline$p$ & $p_{L}$ & $p_{L}\left(\frac{2}{\gamma+1}+\frac{\gamma-1}{\gamma+1} \frac{v_{L}-\xi}{c_{L}}\right)^{\frac{2 \gamma}{\gamma-1}}$ & $p_{R}$ \\
\hline
\end{tabular}

We run Code 1, Code 2, and Code 3 on the computational domain $D=(0,1)$ with $x_{0}=0.2$ and the initial time is $t_{0}=\frac{0.2}{v_{R}-c_{R}}$. The initial data is the exact solution at $t=\frac{0.2}{v_{R}-c_{R}}$ given in Table 2. The simulation are run until $t=0.5$. The consolidated error indicator $\delta_{1}(t)$ defined in (5.1) is reported in Table 3 for Code 1 and Code 2 only for brevity. This series of tests shows that the proposed method, with limitation of the density and the specific entropy as described in $\S 4.4-\S 4.6$, converges with rate at least $\mathcal{O}\left(h^{1.5}\right)$ on the rarefaction wave problem. We observe that the low-order method is indeed asymptotically first-order (the rate is 0.96 for 12800 grid points).

TABLE 3

Rarefaction wave, $\mathbb{P}_{1}$ meshes, Convergence tests with Code 1 and Code 2, CFL=0.25.

\begin{tabular}{|c|c|c|c|c|c|c|c|c|}
\hline \multirow{2}{*}{ \# dofs } & \multicolumn{2}{|c|}{ Code 1 } & \multicolumn{2}{c|}{ Code 2} & \multicolumn{2}{c|}{ Galerkin } & \multicolumn{2}{c|}{ Low-order } \\
\cline { 2 - 9 } & $\delta_{1}(t)$ & rate & $\delta_{1}(t)$ & rate & $\delta_{1}(t)$ & rate & $\delta_{1}(t)$ & rate \\
\hline 100 & $1.01 \mathrm{E}-03$ & & $3.33 \mathrm{E}-03$ & & $1.44 \mathrm{E}-03$ & & $5.10 \mathrm{E}-02$ & \\
200 & $3.28 \mathrm{E}-04$ & 1.62 & $1.08 \mathrm{E}-03$ & 1.61 & $4.38 \mathrm{E}-04$ & 1.71 & $2.96 \mathrm{E}-02$ & 0.78 \\
400 & $1.13 \mathrm{E}-04$ & 1.54 & $3.57 \mathrm{E}-04$ & 1.61 & $1.42 \mathrm{E}-04$ & 1.62 & $1.68 \mathrm{E}-02$ & 0.82 \\
800 & $4.02 \mathrm{E}-05$ & 1.49 & $1.18 \mathrm{E}-04$ & 1.60 & $4.73 \mathrm{E}-05$ & 1.59 & $9.23 \mathrm{E}-03$ & 0.86 \\
1600 & $1.44 \mathrm{E}-05$ & 1.48 & $3.96 \mathrm{E}-05$ & 1.58 & $1.60 \mathrm{E}-05$ & 1.57 & $4.96 \mathrm{E}-03$ & 0.89 \\
3200 & $5.11 \mathrm{E}-06$ & 1.49 & $1.31 \mathrm{E}-05$ & 1.59 & $5.47 \mathrm{E}-06$ & 1.55 & $2.62 \mathrm{E}-03$ & 0.92 \\
6400 & $1.75 \mathrm{E}-06$ & 1.54 & $4.32 \mathrm{E}-06$ & 1.61 & $1.82 \mathrm{E}-06$ & 1.59 & $1.37 \mathrm{E}-03$ & 0.94 \\
12800 & $5.71 \mathrm{E}-07$ & 1.62 & $1.38 \mathrm{E}-06$ & 1.64 & $5.83 \mathrm{E}-07$ & 1.64 & $7.05 \mathrm{E}-04$ & 0.96 \\
\hline
\end{tabular}

When comparing the results from Code 1 with the Galerkin solution, we observe that the extra dissipation induced in Code 1 by the entropy viscosity and limitation is of the order of the truncation error, which is optimal. That is, the method does not introduce any extraneous dissipation on smooth solutions. Note in passing that the maximum norm error indicator $\delta_{\infty}(t)$ has also been computed for this test (not reported here for brevity), yielding the rate $\mathcal{O}(h)$ for Code 1 and $\mathcal{O}\left(h^{0.75}\right)$ for Code 2. The rate $\mathcal{O}(h)$ is optimal since the solution is in $\boldsymbol{W}^{1, \infty}(D)$

5.4. Leblanc shocktube. We continue with a challenging Riemann problem that is known in the literature as the Leblanc shocktube. The data are as follows: $\left(\rho_{L}, v_{L}, p_{L}\right)=\left(1,0,(\gamma-1) 10^{-1}\right)$ and $\left(\rho_{R}, v_{R}, p_{R}\right)=\left(10^{-3}, 0,(\gamma-1) 10^{-10}\right)$ and the equation of state is a gamma-law with $\gamma=\frac{5}{3}$. The exact solution is described in the Table 4. Denoting by $x_{0}$ the location of the discontinuity at $t=0$, the quantity $\xi=\frac{x-x_{0}}{t}$ is the self-similar variable and the other numerical values in the table are given with 15 digit accuracy by $\rho_{L}^{*}=5.40793353493162 \times 10^{-2}, \rho_{R}^{*}=$ 
$3.99999806043000 \times 10^{-3}, v^{*}=0.621838671391735, p *=0.515577927650970 \times 10^{-3}$, $\lambda_{1}=0.495784895188979, \lambda_{3}=0.829118362533470$.

TABLE 4

Solution of the Leblanc shocktube.

\begin{tabular}{|c|c|c|c|c|c|}
\hline & $\xi \leq-\frac{1}{3}$ & $-\frac{1}{3}<\xi \leq \lambda_{1}$ & $\lambda_{1}<\xi \leq v^{*}$ & $v^{*}<\xi \leq \lambda_{3}$ & $\lambda_{3}<\xi$ \\
\hline$\rho$ & $\rho_{L}$ & $(0.75-0.75 \xi)^{3}$ & $\rho_{L}^{*}$ & $\rho_{R}^{*}$ & $\rho_{R}$ \\
\hline$v$ & $v_{L}$ & $0.75\left(\frac{1}{3}+\xi\right)$ & $v^{*}$ & $v^{*}$ & $v_{R}$ \\
\hline$p$ & $p_{L}$ & $\frac{1}{15}(0.75-0.75 \xi)^{5}$ & $p^{*}$ & $p^{*}$ & $p_{R}$ \\
\hline
\end{tabular}

We do simulations with $x_{0}=0.33$ until $t=2 / 3$ with Code 2 and Code 3 . The consolidated error indicator $\delta_{1}(t)$ is reported in Table 5 . The convergence rate on $\delta_{1}(t)$ is clearly first-order which is optimal for this problem.

TABLE 5

Leblanc shocktube, $\mathbb{P}_{1}$ meshes, Convergence tests with Code 1 and Code 2, CFL $=0.25$.

\begin{tabular}{|c|c|c|c|c|c|c|}
\hline \multirow{2}{*}{ \# dofs } & \multicolumn{2}{|c|}{ Code 1} & \multicolumn{2}{c|}{ Code 2} & \multicolumn{2}{c|}{ Low-order } \\
\cline { 2 - 7 } & $\delta_{1}(t)$ & rate & $\delta_{1}(t)$ & rate & $\delta_{1}(t)$ & rate \\
\hline 100 & $1.26 \mathrm{E}-01$ & & $1.49 \mathrm{E}-01$ & & $2.61 \mathrm{E}-01$ & \\
200 & $7.67 \mathrm{E}-02$ & 0.71 & $9.01 \mathrm{E}-02$ & 0.72 & $1.94 \mathrm{E}-01$ & 0.43 \\
400 & $4.31 \mathrm{E}-02$ & 0.83 & $4.92 \mathrm{E}-02$ & 0.87 & $1.41 \mathrm{E}-01$ & 0.46 \\
800 & $2.25 \mathrm{E}-02$ & 0.94 & $2.61 \mathrm{E}-02$ & 0.91 & $9.95 \mathrm{E}-02$ & 0.50 \\
1600 & $1.13 \mathrm{E}-02$ & 0.99 & $1.34 \mathrm{E}-02$ & 0.96 & $6.74 \mathrm{E}-02$ & 0.56 \\
3200 & $5.73 \mathrm{E}-03$ & 0.99 & $6.83 \mathrm{E}-03$ & 0.97 & $4.40 \mathrm{E}-02$ & 0.62 \\
6400 & $2.85 \mathrm{E}-03$ & 1.01 & $3.42 \mathrm{E}-03$ & 0.99 & $2.78 \mathrm{E}-02$ & 0.66 \\
12800 & $1.43 \mathrm{E}-03$ & 1.00 & $1.70 \mathrm{E}-03$ & 1.00 & $1.73 \mathrm{E}-02$ & 0.68 \\
\hline
\end{tabular}

5.5. Sod, Lax, Blast wave. We now illustrate the method on a series of traditional problems without giving the full tables for the convergence rates for brevity. We consider the Sod shocktube, the Lax shocktube, and the Woodward-Collela blast wave. We refer the reader to the literature for the initial data for these test cases. The computations are done on the domain $D=(0,1)$ with CFL $=0.5$ on four different girds with Code 1. The final times are $t=0.225$ for the Sod shocktube, $t=0.15$ for the Lax shocktube, and $t=0.038$ for the Woodward-Collela blast wave. We show the graph of the density for these three cases and for the four meshes in Figure 1. We have observed the convergence rate to be between $\mathcal{O}\left(h^{0.9}\right)$ and $\mathcal{O}(h)$ on $\delta_{1}(t)$ for both the Sod and the Lax shocktubes, which is near optimal (results not reported for brevity).

5.6. 2D isentropic vortex. We now consider a two-dimensional problem introduced in Yee et al. [44]. This test case is often used to assess the accuracy of numerical schemes. The flow field is isentropic; i.e., the solution is smooth and does not involve any steep gradients or discontinuities.

Let $\rho_{\infty}=P_{\infty}=1, \boldsymbol{u}_{\infty}=(2,0)^{\top}$ be free stream values. Let us define the following perturbation values for the velocity and the temperature:

$$
\delta \boldsymbol{u}(\boldsymbol{x}, t)=\frac{\beta}{2 \pi} e^{1-r^{2}}\left(-\bar{x}_{2}, \bar{x}_{1}\right), \quad \delta T(\boldsymbol{x}, t)=-\frac{(\gamma-1) \beta^{2}}{8 \gamma \pi^{2}} e^{1-r^{2}},
$$

where $\beta=5$ is a constant defining the vortex strength, $\gamma=\frac{7}{5}, \overline{\boldsymbol{x}}=\left(x_{1}-x_{1}^{\mathrm{c}}, x_{2}-x_{2}^{\mathrm{c}}\right)$, where $\boldsymbol{x}^{\mathrm{c}}=\left(x_{1}^{\mathrm{c}}+2 t, x_{2}^{\mathrm{c}}\right)$ is the position of the vortex, and $r^{2}=\|\overline{\boldsymbol{x}}\|_{\ell^{2}}^{2}$. The exact 

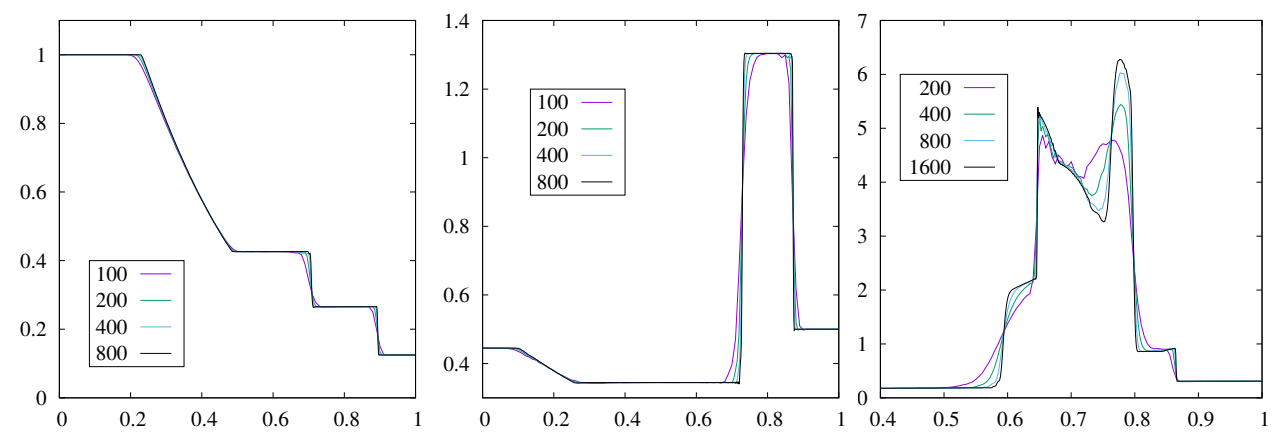

FIG. 1. Code 1, CFL =0.5. Left: Sod shocktube, $t=0.225$; Center: Lax shocktube, $t=0.15$; Right: Woodward-Collela blast wave, $t=0.038$.

solution is a passive convection of the vortex with the mean velocity $\boldsymbol{u}_{\infty}$ :

$$
\rho(\boldsymbol{x}, t)=\left(T_{\infty}+\delta T\right)^{1 / \gamma-1}, \quad \boldsymbol{u}(\boldsymbol{x}, t)=\boldsymbol{u}_{\infty}+\delta \boldsymbol{u}, \quad p(\boldsymbol{x}, t)=\rho^{\gamma} .
$$

We perform the numerical computation in the rectangle $D=(-5,10) \times(-5,5)$ from $t=0$ until $t=2$. The initial mesh consists of $20 \times 13$ squares divided by four equilateral triangles, then the mesh is refined uniformly to compute finer solutions. The computations are done with Code 3 . The consolidated error indicator $\delta_{\infty}(t)$ is reported in Table 6 . Here again we observe second-order accuracy in the maximum norm.

TABLE 6

Isentropic vortex, $\mathbb{P}_{1}$ meshes, convergence tests, $t=2$. Code $3, C F L=0.5$.

\begin{tabular}{|c|c|c|}
\hline \# dofs & $\delta_{\infty}(t)$ & rate \\
\hline 2216 & $2.23 \mathrm{e}-01$ & - \\
8588 & $4.50 \mathrm{e}-02$ & 2.37 \\
34456 & $1.05 \mathrm{e}-02$ & 2.09 \\
136748 & $2.82 \mathrm{e}-03$ & 1.91 \\
547416 & $9.09 \mathrm{e}-04$ & 1.63 \\
\hline
\end{tabular}

5.7. Mach 3 step. We finish by illustrating the method on the classical Mach 3 flow in a wind tunnel with a forward facing step. The computational domain is $D=(0,1) \times(0,3) \backslash(0.6,3) \times(0,0.2)$; the geometry of the domain is shown in Figure 2 . The initial data is $\rho=1.4, p=1, \boldsymbol{v}=(3,0)^{\mathrm{\top}}$. The inflow boundary conditions are $\rho_{\mid\{x=0\}}=1.4, p_{\mid\{x=0\}}=1, \boldsymbol{v}_{\mid\{x=0\}}=(3,0)^{\top}$. The outflow boundary conditions are free, i.e., we do nothing at $\{x=3\}$. On the top and bottom boundaries of the channel we enforce $\boldsymbol{v} \cdot \boldsymbol{n}=0$. This is done by setting $\boldsymbol{m}_{i}^{n+1} \cdot \boldsymbol{n}=0$ at the end of each substep of the SSP RK(3,3) algorithm at each node $\boldsymbol{x}_{i}$ belonging to the boundary in question; moreover the vectors $\boldsymbol{c}_{i j}$ introduced in (2.10) are redefined as follows $\boldsymbol{c}_{i j}=-\int_{D} \varphi_{j} \nabla \varphi_{i} \mathrm{~d} x$ at each node $\boldsymbol{x}_{i}$ belonging to the boundary in question. This integration by parts is justified by the observation that it implies global conservation under the assumption that $\boldsymbol{f}(\boldsymbol{u}) \cdot \boldsymbol{n}=0$ over the entire boundary of $D$.

The computation is done from $t=0$ to $t=4$. We show in Figure 2 a Schlierentype snapshot of the density at $t=4$ obtained with Code 2 . The Kelvin-Helmholtz instability of the contact discontinuity is clearly visible. No regularization or smoothing is applied at the top left corner of the step. 


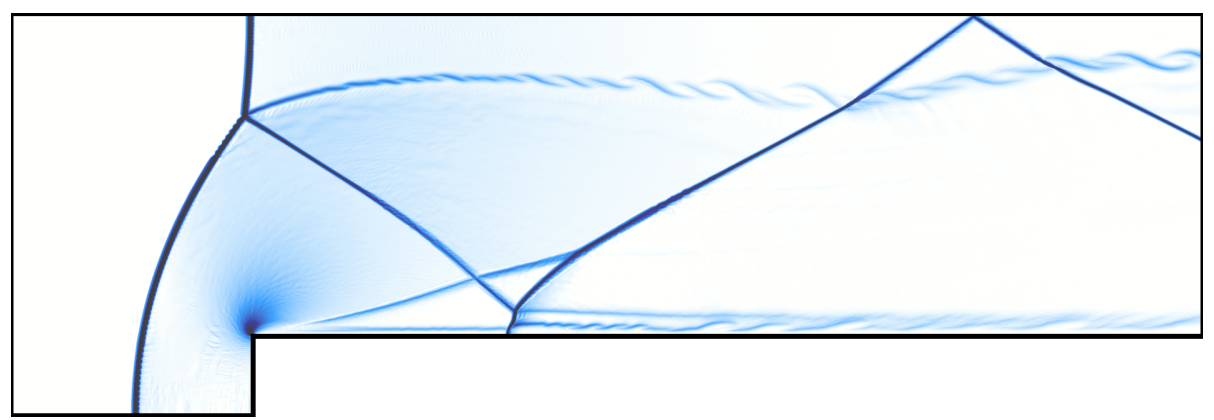

FIG. 2. Mach 3 step, density, $t=4$, density, $\mathbb{P}_{1}$ approximation using Code 2.

\section{References.}

[1] D. Arndt, W. Bangerth, D. Davydov, T. Heister, L. Heltai, M. Kronbichler, M. Maier, J.-P. Pelteret, B. Turcksin, and D. Wells. The deal. II library, version 8.5. Journal of Numerical Mathematics, 2017. doi: 10.1515/jnma-2016-1045.

[2] W. Bangerth, R. Hartmann, and G. Kanschat. deal.II - a general purpose object oriented finite element library. ACM Trans. Math. Softw., 33(4):24/1-24/27, 2007.

[3] M. Berger, M. J. Aftosmis, and S. M. Murman. Analysis of slope limiters on irregular grids. AIAA Paper 2005-0490, American Institute for Aeronautics and Astronautics, Reno, NV, USA, May 2005. Also NASA TM NAS-05-007.

[4] J. P. Boris and D. L. Book. Flux-corrected transport. I. SHASTA, a fluid transport algorithm that works [J. Comput. Phys. 11 (1973), no. 1, 38-69]. J. Comput. Phys., 135(2):170-186, 1997. With an introduction by Steven T. Zalesak, Commemoration of the 30th anniversary of J. Comput. Phys.

[5] M. A. Christon, M. J. Martinez, and T. E. Voth. Generalized Fourier analyses of the advection-diffusion equation-part I: one-dimensional domains. International Journal for Numerical Methods in Fluids, 45(8):839-887, 2004.

[6] K. N. Chueh, C. C. Conley, and J. A. Smoller. Positively invariant regions for systems of nonlinear diffusion equations. Indiana Univ. Math. J., 26(2):373-392, 1977.

[7] H. Frid. Maps of convex sets and invariant regions for finite-difference systems of conservation laws. Arch. Ration. Mech. Anal., 160(3):245-269, 2001.

[8] E. Godlewski and P.-A. Raviart. Numerical approximation of hyperbolic systems of conservation laws, volume 118 of Applied Mathematical Sciences. SpringerVerlag, New York, 1996.

[9] P. Gresho, R. Sani, and M. Engelman. Incompressible flow and the finite element method: advection-diffusion and isothermal laminar flow. Incompressible Flow \& the Finite Element Method. Wiley, 1998.

[10] J.-L. Guermond and R. Pasquetti. A correction technique for the dispersive effects of mass lumping for transport problems. Comput. Methods Appl. Mech. Engrg., 253:186-198, 2013.

[11] J.-L. Guermond and B. Popov. Invariant domains and first-order continuous finite element approximation for hyperbolic systems. SIAM J. Numer. Anal., 54 (4):2466-2489, 2016.

[12] J.-L. Guermond and B. Popov. Fast estimation from above of the maximum wave speed in the Riemann problem for the Euler equations. J. Comput. Phys., 
321:908-926, 2016.

[13] J.-L. Guermond and B. Popov. Invariant domains and second-order continuous finite element approximation for scalar conservation equations. SIAM J. on Numer. Anal., 2016, in review.

[14] J.-L. Guermond and B. Popov. Second-order positivity preserving continuous finite element approximation of hyperbolic systems of conservation laws. 2017, preprint.

[15] J.-L. Guermond, R. Pasquetti, and B. Popov. Entropy viscosity method for nonlinear conservation laws. J. Comput. Phys., 230(11):4248-4267, 2011.

[16] J.-L. Guermond, M. Nazarov, B. Popov, and Y. Yang. A second-order maximum principle preserving Lagrange finite element technique for nonlinear scalar conservation equations. SIAM J. Numer. Anal., 52(4):2163-2182, 2014.

[17] A. Harten. On the symmetric form of systems of conservation laws with entropy. J. Comput. Phys., 49(1):151-164, 1983.

[18] A. Harten and S. Osher. Uniformly high-order accurate nonoscillatory schemes. I. SIAM J. Numer. Anal., 24(2):279-309, 1987.

[19] A. Harten, P. D. Lax, C. D. Levermore, and W. J. Morokoff. Convex entropies and hyperbolicity for general Euler equations. SIAM J. Numer. Anal., 35(6): 2117-2127 (electronic), 1998.

[20] J. S. Hesthaven. From electrostatics to almost optimal nodal sets for polynomial interpolation in a simplex. SIAM J. Numer. Anal., 35(2):655-676, 1998.

[21] D. Hoff. Invariant regions for systems of conservation laws. Trans. Amer. Math. Soc., 289(2):591-610, 1985.

[22] A. Jameson. Origins and further development of the Jameson-Schmidt-Turkel scheme. AIAA Journal, 55(5), 2017.

[23] A. Jameson, W. Schmidt, and E. Turkel. Numerical solution of the Euler equations by finite volume. methods using Runge-Kutta time-stepping schemes. In 14th AIAA Fluid and Plasma Dynamics Conference, June 1981. AIAA Paper $1981-1259$.

[24] Y. Jiang and H. Liu. An invariant-region-preserving (irp) limiter for compressible euler equations. In Proceedings of the Hyp2016 conference, 2017.

[25] B. Khobalatte and B. Perthame. Maximum principle on the entropy and secondorder kinetic schemes. Math. Comp., 62(205):119-131, 1994.

[26] J. F. B. M. Kraaijevanger. Contractivity of Runge-Kutta methods. BIT, 31(3): 482-528, 1991.

[27] D. Kuzmin and M. Möller. Algebraic flux correction. II. Compressible Euler equations. In Flux-corrected transport, Sci. Comput., pages 207-250. Springer, Berlin, 2005.

[28] D. Kuzmin and S. Turek. Flux correction tools for finite elements. Journal of Computational Physics, 175(2):525-558, 2002.

[29] D. Kuzmin, R. Löhner, and S. Turek. Flux-Corrected Transport. Scientific Computation. Springer, 2005. 3-540-23730-5.

[30] A. Logg, K.-A. Mardal, G. N. Wells, et al. Automated Solution of Differential Equations by the Finite Element Method. Springer, 2012.

[31] C. Lohmann and D. Kuzmin. Synchronized flux limiting for gas dynamics variables. Journal of Computational Physics, 326:973 - 990, 2016.

[32] R. Löhner, K. Morgan, J. Peraire, and M. Vahdati. Finite element flux-corrected transport (femfct) for the euler and navierstokes equations. International Journal for Numerical Methods in Fluids, 7(10):1093-1109, 1987.

[33] O. Metayer and R. Saurel. The noble-abel stiffened-gas equation of state. Phys. 
Fluids, 28(4):046102, 2016.

[34] H. Nessyahu and E. Tadmor. Nonoscillatory central differencing for hyperbolic conservation laws. J. Comput. Phys., 87(2):408-463, 1990.

[35] B. Perthame and Y. Qiu. A variant of Van Leer's method for multidimensional systems of conservation laws. J. Comput. Phys., 112(2):370-381, 1994.

[36] B. Perthame and C.-W. Shu. On positivity preserving finite volume schemes for Euler equations. Numer. Math., 73(1):119-130, 1996.

[37] B. Schmidtmann, R. Abgrall, and M. Torrilhon. On third-order limiter functions for finite volume methods. Bull. Braz. Math. Soc. (N.S.), 47(2):753-764, 2016.

[38] D. Serre. Systems of conservation laws. 2. Cambridge University Press, Cambridge, 2000. Geometric structures, oscillations, and initial-boundary value problems, Translated from the 1996 French original by I. N. Sneddon.

[39] C.-W. Shu and S. Osher. Efficient implementation of essentially non-oscillatory shock-capturing schemes. J. Comput. Phys., 77(2):439 - 471, 1988.

[40] M. A. Taylor, B. A. Wingate, and R. E. Vincent. An algorithm for computing Fekete points in the triangle. SIAM J. Numer. Anal., 38(5):1707-1720, 2000.

[41] T. Thompson. A discrete commutator theory for the consistency and phase error analysis of semi-discrete $C^{0}$ finite element approximations to the linear transport equation. J. Comput. Appl. Math., 303:229-248, 2016.

[42] E. F. Toro. Riemann solvers and numerical methods for fluid dynamics. SpringerVerlag, Berlin, third edition, 2009. A practical introduction.

[43] T. Warburton. An explicit construction of interpolation nodes on the simplex. J. Engrg. Math., 56(3):247-262, 2006.

[44] H. C. Yee, N. D. Sandham, and M. J. Djomehri. Low-dissipative high-order shock-capturing methods using characteristic-based filters. J. Comput. Phys., 150(1):199-238, 1999.

[45] S. T. Zalesak. Fully multidimensional flux-corrected transport algorithms for fluids. J. Comput. Phys., 31(3):335-362, 1979.

[46] S. T. Zalesak. The design of flux-corrected transport (FCT) algorithms for structured grids. In Flux-corrected transport, Sci. Comput., pages 29-78. Springer, Berlin, 2005.

[47] X. Zhang and C.-W. Shu. On positivity-preserving high order discontinuous Galerkin schemes for compressible Euler equations on rectangular meshes. $J$. Comput. Phys., 229(23):8918-8934, 2010.

[48] X. Zhang and C.-W. Shu. A minimum entropy principle of high order schemes for gas dynamics equations. Numer. Math., 121(3):545-563, 2012. 